\title{
Events with isolated leptons and missing transverse momentum and measurement of $W$ production at HERA
}

\section{The H1 Collaboration}

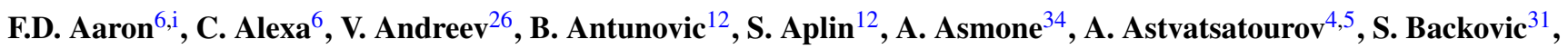

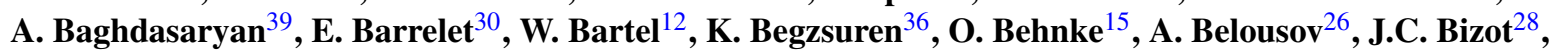
V. Boudry ${ }^{29}$, I. Bozovic-Jelisavcic ${ }^{2}$, J. Bracinik ${ }^{3}$, G. Brandt ${ }^{12}$, M. Brinkmann ${ }^{12}$, V. Brisson ${ }^{28}$, D. Bruncko ${ }^{17}$, A. Bunyatyan ${ }^{14,39}$, G. Buschhorn ${ }^{27}$, L. Bystritskaya ${ }^{25}$, A.J. Campbell ${ }^{12}$, K.B. Cantun Avila ${ }^{23}$, F. Cassol-Brunner ${ }^{22}$, K. Cerny ${ }^{33}$, V. Cerny ${ }^{17, g}$, V. Chekelian ${ }^{27}$, A. Cholewa ${ }^{12}$, J.G. Contreras ${ }^{23}$, J.A. Coughlan ${ }^{7}$, G. Cozzika ${ }^{11}$, J. Cvach $^{32}$, J.B. Dainton ${ }^{19}$, K. Daum ${ }^{38, c}$, M. Deák ${ }^{12}$, Y. de Boer ${ }^{12}$, B. Delcourt ${ }^{28}$, M. Del Degan ${ }^{41}$, J. Delvax ${ }^{4,5}$, A. De Roeck ${ }^{12, e}$,

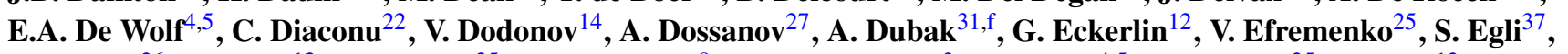
A. Eliseev ${ }^{26}$, E. Elsen ${ }^{12}$, S. Essenov ${ }^{25}$, A. Falkiewicz ${ }^{8}$, P.J.W. Faulkner ${ }^{3}$, L. Favart ${ }^{4,5}$, A. Fedotov ${ }^{25}$, R. Felst ${ }^{12}$, J. Feltesse ${ }^{11, \mathrm{~h}}$, J. Ferencei ${ }^{17}$, D.-J. Fischer ${ }^{12}$, M. Fleischer ${ }^{12}$, A. Fomenko ${ }^{26}$, E. Gabathuler ${ }^{19}$, J. Gayler ${ }^{12}$, S. Ghazaryan ${ }^{39}$, A. Glazov ${ }^{12}$, I. Glushkov ${ }^{40}$, L. Goerlich ${ }^{8}$, M. Goettlich ${ }^{13}$, N. Gogitidze ${ }^{26}$, M. Gouzevitch ${ }^{29}$, C. Grab ${ }^{41}$, T. Greenshaw ${ }^{19}$, B.R. Grell ${ }^{12}$, G. Grindhammer ${ }^{27}$, S. Habib ${ }^{13, j}$, D. Haidt ${ }^{12}$, M. Hansson ${ }^{21}$, C. Helebrant $^{12}$, R.C.W. Henderson ${ }^{18}$, E. Hennekemper ${ }^{16}$, H. Henschel ${ }^{40}$, G. Herrera ${ }^{24}$, M. Hildebrandt ${ }^{37}$, K.H. Hiller ${ }^{40}$, D. Hoffmann ${ }^{22}$, R. Horisberger ${ }^{37}$, T. Hreus ${ }^{4,5, \mathrm{~d}}$, M. Jacquet ${ }^{28}$, M.E. Janssen ${ }^{12}$, X. Janssen ${ }^{4,5}$, V. Jemanov ${ }^{13}$, L. Jönsson ${ }^{21}$, A.W. Jung ${ }^{16}$, H. Jung ${ }^{12}$, M. Kapichine ${ }^{10}$, J. Katzy ${ }^{12}$, I.R. Kenyon ${ }^{3}$, C. Kiesling ${ }^{27}$, M. Klein ${ }^{19}$, C. Kleinwort ${ }^{12}$, T. Klimkovich ${ }^{12}$, T. Kluge ${ }^{19}$, A. Knutsson ${ }^{12}$, R. Kogler ${ }^{27}$, V. Korbel ${ }^{12}$, P. Kostka ${ }^{40}$, M. Kraemer ${ }^{12}$, K. Krastev ${ }^{12}$, J. Kretzschmar ${ }^{19}$, A. Kropivnitskaya ${ }^{25}$, K. Krüger ${ }^{16}$, K. Kutak ${ }^{12}$, M.P.J. Landon ${ }^{20}$, W. Lange ${ }^{40}$, G. Laštovička-Medin ${ }^{31}$, P. Laycock ${ }^{19}$, A. Lebedev ${ }^{26}$, G. Leibenguth ${ }^{41}$, V. Lendermann ${ }^{16}$, S. Levonian ${ }^{12}$,

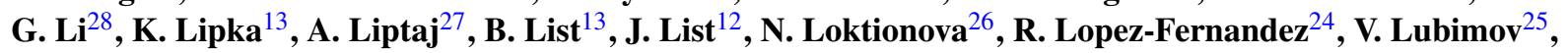
L. Lytkin ${ }^{14}$, A. Makankine ${ }^{10}$, E. Malinovski ${ }^{26}$, P. Marage $^{4,5}$, Ll. Marti $^{12}$, H.-U. Martyn ${ }^{1}$, S.J. Maxfield ${ }^{19}$, A. Mehta ${ }^{19}$, K. Meier ${ }^{16}$, A.B. Meyer $^{12}$, H. Meyer ${ }^{12}$, H. Meyer ${ }^{38}$, J. Meyer ${ }^{12}$, V. Michels ${ }^{12}$, S. Mikocki ${ }^{8}$, I. Milcewicz-Mika ${ }^{8}$, F. Moreau $^{29}$, A. Morozov ${ }^{10}$, J.V. Morris ${ }^{7}$, M.U. Mozer ${ }^{4,5}$, M. Mudrinic ${ }^{2}$, K. Müller ${ }^{42}$, P. Murín ${ }^{17, d}$, B. Naroska ${ }^{13, \dagger}$, Th. Naumann ${ }^{40}$, P.R. Newman ${ }^{3}$, C. Niebuhr ${ }^{12}$, A. Nikiforov ${ }^{12}$, G. Nowak ${ }^{8}$, K. Nowak ${ }^{42}$, M. Nozicka ${ }^{12}$, B. Olivier ${ }^{27}$, J.E. Olsson ${ }^{12}$, S. Osman ${ }^{21}$, D. Ozerov ${ }^{25}$, V. Palichik ${ }^{10}$, I. Panagoulias ${ }^{12, b, w}$, M. Pandurovic ${ }^{2}$, Th. Papadopoulou ${ }^{12, b, w}$, C. Pascaud $^{28}$, G.D. Patel ${ }^{19}$, O. Pejchal ${ }^{33}$, E. Perez ${ }^{11, e}$, A. Petrukhin ${ }^{25}$, I. Picuric ${ }^{31}$, S. Piec $^{40}$, D. Pitzl ${ }^{12}$,

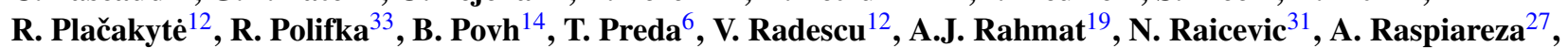

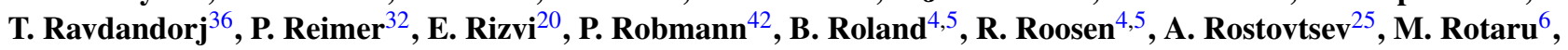
J.E. Ruiz Tabasco ${ }^{23}$, Z. Rurikova ${ }^{12}$, S. Rusakov ${ }^{26}$, D. Śálek $^{33}$, D.P.C. Sankey ${ }^{7}$, M. Sauter ${ }^{41}$, E. Sauvan ${ }^{22}$, S. Schmitt ${ }^{12}$, C. Schmitz ${ }^{42}$, L. Schoeffel ${ }^{11}$, A. Schöning ${ }^{12,42, a}$, H.-C. Schultz-Coulon ${ }^{16}$, F. Sefkow ${ }^{12}$, R.N. Shaw-West ${ }^{3}$, I. Sheviakov ${ }^{26}$, L.N. Shtarkov ${ }^{26}$, S. Shushkevich ${ }^{27}$, T. Sloan ${ }^{18}$, I. Smiljanic ${ }^{2}$, Y. Soloviev ${ }^{26}$, P. Sopicki ${ }^{8}$, D. South ${ }^{9}$, V. Spaskov ${ }^{10}$, A. Specka ${ }^{29}$, Z. Staykova ${ }^{12}$, M. Steder ${ }^{12}$, B. Stella ${ }^{34}$, G. Stoicea ${ }^{6}$, U. Straumann ${ }^{42}$, D. Sunar ${ }^{4,5}$, T. Sykora ${ }^{4,5}$, V. Tchoulakov ${ }^{10}$, G. Thompson ${ }^{20}$, P.D. Thompson ${ }^{3}$, T. Toll ${ }^{12}$, F. Tomasz ${ }^{17}$, T.H. Tran $^{28}$, D. Traynor $^{20}$,

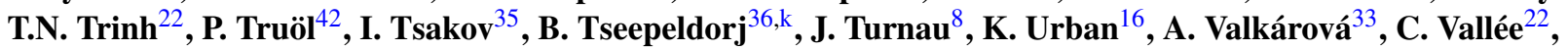
P. Van Mechelen ${ }^{4,5}$, A. Vargas Trevino ${ }^{12}$, Y. Vazdik ${ }^{26}$, S. Vinokurova ${ }^{12}$, V. Volchinski ${ }^{39}$, D. Wegener ${ }^{9}$, Ch. Wissing ${ }^{12}$, E. Wünsch ${ }^{12}$, J. Žáček ${ }^{33}$, J. Zálešák ${ }^{32}$, Z. Zhang ${ }^{28}$, A. Zhokin ${ }^{25}$, T. Zimmermannn ${ }^{41}$, H. Zohrabyan ${ }^{39}$, F. Zomer ${ }^{28}$

\footnotetext{
${ }^{1}$ I. Physikalisches Institut der RWTH, Aachen, Germany ${ }^{1}$

${ }^{2}$ Vinca Institute of Nuclear Sciences, Belgrade, Serbia

${ }^{3}$ School of Physics and Astronomy, University of Birmingham, Birmingham, $\mathrm{UK}^{\mathrm{m}}$

${ }^{4}$ Inter-University Institute for High Energies ULB-VUB, Brussels, Belgium

${ }^{5}$ Universiteit Antwerpen, Antwerpen, Belgium $^{\mathrm{n}}$

${ }^{6}$ National Institute for Physics and Nuclear Engineering (NIPNE), Bucharest, Romania

${ }^{7}$ Rutherford Appleton Laboratory, Chilton, Didcot, $\mathrm{UK}^{\mathrm{m}}$

${ }^{8}$ Institute for Nuclear Physics, Cracow, Poland ${ }^{\circ}$

${ }^{9}$ Institut für Physik, TU Dortmund, Dortmund, Germany ${ }^{1}$

${ }^{10}$ Joint Institute for Nuclear Research, Dubna, Russia
} 
${ }^{11} \mathrm{CEA}, \mathrm{DSM} / \mathrm{Irfu}, \mathrm{CE}-$ Saclay, Gif-sur-Yvette, France

${ }^{12}$ DESY, Hamburg, Germany

${ }^{13}$ Institut für Experimentalphysik, Universität Hamburg, Hamburg, Germany ${ }^{1}$

${ }^{14}$ Max-Planck-Institut für Kernphysik, Heidelberg, Germany

${ }^{15}$ Physikalisches Institut, Universität Heidelberg, Heidelberg, Germany ${ }^{1}$

${ }^{16}$ Kirchhoff-Institut für Physik, Universität Heidelberg, Heidelberg, Germany ${ }^{1}$

${ }^{17}$ Institute of Experimental Physics, Slovak Academy of Sciences, Košice, Slovak Republic ${ }^{q}$

${ }^{18}$ Department of Physics, University of Lancaster, Lancaster, UK $^{\mathrm{m}}$

${ }^{19}$ Department of Physics, University of Liverpool, Liverpool, $\mathrm{UK}^{\mathrm{m}}$

${ }^{20}$ Queen Mary and Westfield College, London, $\mathrm{UK}^{\mathrm{m}}$

${ }^{21}$ Physics Department, University of Lund, Lund, Sweden ${ }^{\mathrm{r}}$

${ }^{22}$ CPPM, CNRS/IN2P3, Univ. Mediterranee, Marseille, France

${ }^{23}$ Departamento de Fisica Aplicada, CINVESTAV, Mérida, Yucatán, Mexico ${ }^{u}$

${ }^{24}$ Departamento de Fisica, CINVESTAV IPN, Mexico City, Mexico ${ }^{\mathrm{u}}$

${ }^{25}$ Institute for Theoretical and Experimental Physics, Moscow, Russiav

${ }^{26}$ Lebedev Physical Institute, Moscow, Russia ${ }^{p}$

${ }^{27}$ Max-Planck-Institut für Physik, München, Germany

${ }^{28}$ LAL, Univ Paris-Sud, CNRS/IN2P3, Orsay, France

${ }^{29}$ LLR, Ecole Polytechnique, IN2P3-CNRS, Palaiseau, France

${ }^{30}$ LPNHE, Universités Paris VI and VII, IN2P3-CNRS, Paris, France

${ }^{31}$ Faculty of Science, University of Montenegro, Podgorica, Montenegro ${ }^{p}$

${ }^{32}$ Institute of Physics, Academy of Sciences of the Czech Republic, Praha, Czech Republic ${ }^{\mathrm{s}}$

${ }^{33}$ Faculty of Mathematics and Physics, Charles University, Praha, Czech Republic ${ }^{\mathrm{s}}$

${ }_{35}^{34}$ Dipartimento di Fisica, Università di Roma Tre and INFN Roma 3, Roma, Italy

${ }^{35}$ Institute for Nuclear Research and Nuclear Energy, Sofia, Bulgaria ${ }^{\mathrm{p}}$

${ }^{36}$ Institute of Physics and Technology of the Mongolian Academy of Sciences, Ulaanbaatar, Mongolia

${ }^{37}$ Paul Scherrer Institut, Villigen, Switzerland

${ }^{38}$ Fachbereich C, Universität Wuppertal, Wuppertal, Germany

${ }^{39}$ Yerevan Physics Institute, Yerevan, Armenia

${ }^{40}$ DESY, Zeuthen, Germany

${ }^{41}$ Institut für Teilchenphysik, ETH, Zürich, Switzerland ${ }^{t}$

${ }^{42}$ Physik-Institut der Universität Zürich, Zürich, Switzerland ${ }^{t}$

Received: 5 January 2009 / Revised: 1 September 2009 / Published online: 8 October 2009

(C) Springer-Verlag / Società Italiana di Fisica 2009

a e-mail: schoning@mail.desy.de

${ }^{b}$ Also at Physics Department, National Technical University, Zografou Campus, 15773 Athens, Greece.

${ }^{\mathrm{c}}$ Also at Rechenzentrum, Universität Wuppertal, Wuppertal, Germany.

${ }^{\mathrm{d}}$ Also at University of P.J. Šafárik, Košice, Slovak Republic.

${ }^{\mathrm{e}}$ Also at CERN, Geneva, Switzerland.

${ }^{\mathrm{f}}$ Also at Max-Planck-Institut für Physik, München, Germany.

${ }^{g}$ Also at Comenius University, Bratislava, Slovak Republic.

${ }^{\mathrm{h}}$ Also at DESY and University Hamburg, Helmholtz Humboldt Research Award.

${ }^{\mathrm{i}}$ Also at Faculty of Physics, University of Bucharest, Bucharest, Romania.

${ }^{j}$ Supported by a scholarship of the World Laboratory Björn Wiik Research Project.

${ }^{\mathrm{k}}$ Also at Ulaanbaatar University, Ulaanbaatar, Mongolia.

${ }^{\dagger}$ Deceased.

${ }^{1}$ Supported by the Bundesministerium für Bildung und Forschung, FRG, under contract numbers $05 \mathrm{H} 1$ 1GUA /1, $05 \mathrm{H} 1$ 1PAA /1, 05 $\mathrm{H} 1$ 1PAB /9, $05 \mathrm{H} 1$ 1PEA /6, $05 \mathrm{H} 1$ 1VHA /7 and $05 \mathrm{H} 1$ 1VHB $/ 5$.

${ }^{\mathrm{m}}$ Supported by the UK Science and Technology Facilities Council, and formerly by the UK Particle Physics and Astronomy Research Council.
Abstract Events with high energy isolated electrons, muons or tau leptons and missing transverse momentum are studied using the full $e^{ \pm} p$ data sample collected by the H1 experiment at HERA, corresponding to an integrated luminosity of $474 \mathrm{pb}^{-1}$. Within the Standard Model, events with

${ }^{\mathrm{n}}$ Supported by FNRS-FWO-Vlaanderen, IISN-IIKW and IWT and by Interuniversity Attraction Poles Programme, Belgian Science Policy.

${ }^{\circ}$ Partially Supported by Polish Ministry of Science and Higher Education, grant PBS/DESY/70/2006.

PSupported by the Deutsche Forschungsgemeinschaft.

${ }^{\mathrm{q}}$ Supported by VEGA SR grant No. 2/7062/ 27.

${ }^{\mathrm{r}}$ Supported by the Swedish Natural Science Research Council.

${ }^{\text {s }}$ Supported by the Ministry of Education of the Czech Republic under the projects LC527, INGO-1P05LA259 and MSM0021620859.

${ }^{\mathrm{t}}$ Supported by the Swiss National Science Foundation.

"Supported by CONACYT, México, grant 48778-F.

v Russian Foundation for Basic Research (RFBR), grant No. 1329.2008.2.

${ }^{w}$ This project is co-funded by the European Social Fund $(75 \%)$ and National Resources (25\%), EPEAEK II, PYTHAGORAS II. 
isolated leptons and missing transverse momentum mainly originate from the production of single $W$ bosons. The total single $W$ boson production cross section is measured as $1.14 \pm 0.25$ (stat.) \pm 0.14 (sys.) pb, in agreement with the Standard Model expectation. The data are also used to establish limits on the $W W \gamma$ gauge couplings and for a measurement of the $W$ boson polarisation.

\section{Introduction}

Events containing high energy leptons and missing transverse momentum produced in high energy particle collisions are interesting as they may be a signature of physics beyond the Standard Model (SM). Such events have been observed by the H1 Collaboration in ep collisions at HERA [1,2]. In the SM, the production of single $W$ bosons with subsequent leptonic decay gives rise to this topology. An excess of electron ${ }^{1}$ and muon events with large missing transverse momentum containing a hadronic final state at high transverse momentum $P_{T}^{X}$ was previously reported by $\mathrm{H} 1$ using $105 \mathrm{pb}^{-1}$ of $e^{+} p$ data [3]. In the region $P_{T}^{X}>25 \mathrm{GeV}$ ten events were observed compared to a SM prediction of $2.9 \pm 0.5$. This observation inspired searches for anomalous single top production [4-6] and bosonic stop decays in $R$ parity violating SUSY [7] and has motivated further possible interpretations [8-11]. The observed excess of events over the SM prediction was not confirmed by the ZEUS Collaboration $[5,6,12]$. In this paper the complete $\mathrm{H} 1$ data sample, collected in the period 1994-2007, is analysed.

The search for isolated tau leptons complements the analysis of the electron and muon channels. If lepton universality holds, the same rate of tau leptons is expected from SM processes. Moreover, an enhanced rate of tau leptons is expected in many new physics scenarios [9]. The search for events containing high energy tau leptons and missing transverse momentum, where the tau is identified by its hadronic decay, has previously been performed by the ZEUS and H1 experiments [13, 14]. ZEUS reported two tau candidates at high $P_{T}^{X}>25 \mathrm{GeV}$, where only $0.2 \pm 0.05$ were predicted from the SM. No events with large hadronic transverse momentum were observed in the $\mathrm{H} 1$ data.

As the SM expectation in the cleaner electron and muon channels is dominated by single $W$ production, this cross section is determined. This measurement is also used to constrain coupling parameters of the $W W \gamma$ vertex. The polarisation fractions of the $W$ boson are also measured for the first time at HERA.

\footnotetext{
${ }^{1}$ In this paper the term "electron" is used generically to refer to both electrons and positrons, if not otherwise stated.
}

\section{Standard model processes and their simulation}

In the search for events with isolated leptons and missing transverse momentum, processes are considered signal if they produce events containing a genuine isolated lepton and genuine missing transverse momentum in the final state. All other processes are defined as background and contribute to the selected sample through misidentification or mismeasurement. Studies on how background processes enter the sample are discussed in Appendix A.

Single $W$ boson production in $e p$ collisions with subsequent leptonic decay $W \rightarrow \ell v$, as illustrated in Figs. 1(a)(c), is the main SM process that produces events with high energy isolated leptons and missing transverse momentum. The SM prediction for $W$ production via $e p \rightarrow e W^{ \pm} X$ is calculated in the framework of the EPVEC event generator [15], which employs the full set of LO diagrams, including $W$ production via the $W W \gamma$ triple gauge boson coupling as illustrated in Fig. 1(b). Each event generated by EPVEC according to its LO cross section is weighted by a factor dependent on the transverse momentum and rapidity of the $W$, such that the resulting cross section corresponds to the NLO calculation [16-19]. The ACFGP [20] parameterisation is used for the photon structure and the CTEQ4M [21] parton distribution functions are used for the proton. The renormalisation scale is taken to be equal to the factorisation scale and is fixed to the $W$ mass. Final state parton showers are simulated using the PYTHIA framework [22]. The NLO corrections are found to be of the order of $30 \%$ at low $W$ transverse momentum (resolved photon interactions) and typically $10 \%$ at high $W$ transverse momentum (direct photon interactions) [16-19]. The NLO calculation reduces the theory error to $15 \%$, compared to $30 \%$ at leading order. The charged current $W$ production process $e p \rightarrow v W^{ \pm} X$, illustrated in Fig. 1(c), is calculated at LO with EPVEC and found to contribute only $7 \%$ of the predicted signal cross section. The total $W$ production cross section, calculated in this way, amounts to $1.1 \mathrm{pb}$ for an electron-proton centre of mass energy of $\sqrt{s}=301 \mathrm{GeV}$ and $1.3 \mathrm{pb}$ for $\sqrt{s}=319 \mathrm{GeV}$.

Signal events may also arise from $Z$ production with subsequent decay to neutrinos. The outgoing electron from this reaction is the isolated lepton in the event, while genuine missing transverse momentum is produced by the neutrinos. This process, illustrated in Fig. 1(d), is also calculated with the EPVEC generator and found to contribute less than 3\% of the predicted signal cross section.

The SM background processes that may mimic the signature through misidentification or mismeasurement are neutral current (NC) and charged current (CC) deep-inelastic scattering (DIS), photoproduction, lepton pair production and photons from wide angle bremsstrahlung. The RAPGAP [23] event generator, which implements the Born level, 
Fig. 1 Diagrams of processes at HERA with an isolated lepton and missing transverse momentum in the final state: (a) $e p \rightarrow e W^{ \pm}(\rightarrow \ell v) X$; (b) $W$ production via the $W W \gamma$ triple gauge boson coupling; (c) $e p \rightarrow v W^{ \pm}(\rightarrow \ell v) X$; (d) $e p \rightarrow e Z(\rightarrow v \bar{v}) X$. The diagrams are shown for $e^{+} p$ collisions

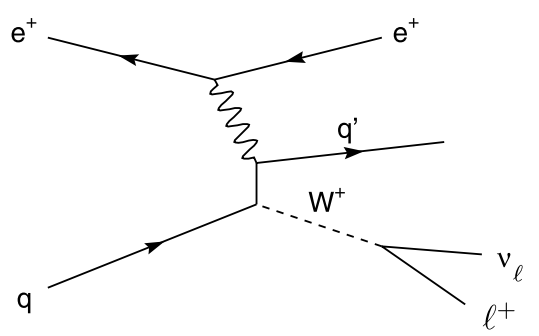

(a)

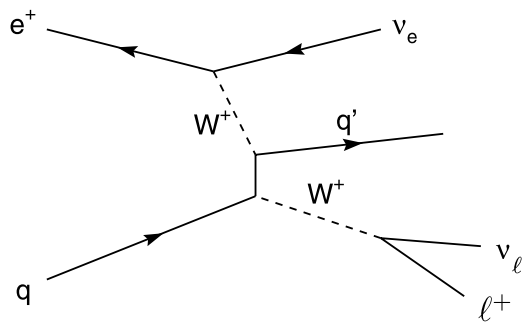

(c)

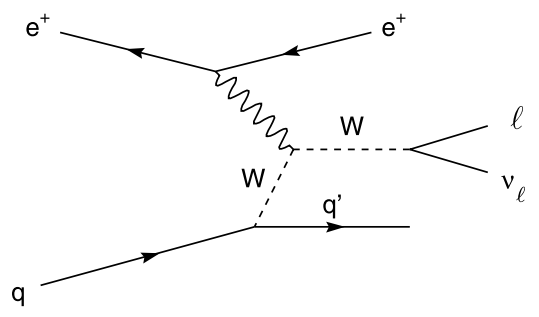

(b)

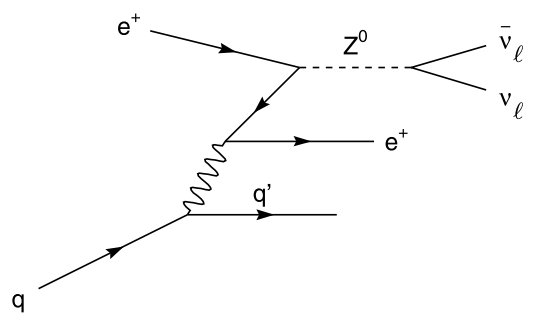

(d)
QCD Compton and boson gluon fusion matrix elements, is used to model NC DIS events. The QED radiative effects arising from real photon emission from both the incoming and outgoing electrons are simulated using the HERACLES [24] program. Contributions from elastic and quasielastic QED Compton scattering are simulated with the WABGEN [25] generator. Direct and resolved photoproduction of jets and prompt photon production are simulated using the PYTHIA [26] event generator. The simulation is based on Born level hard scattering matrix elements with radiative QED corrections. In RAPGAP and PYTHIA, jet production from higher order QCD radiation is simulated using leading logarithmic parton showers and hadronisation is modelled with Lund string fragmentation. The leading order prediction for NC DIS and photoproduction processes with two or more high transverse momentum jets is scaled by a factor of 1.2, to account for higher order QCD corrections to the Monte Carlo (MC) generators [27, 28]. Charged current DIS events are simulated using the DJANGO [29] generator, which includes first order leptonic QED radiative corrections based on HERACLES. The production of two or more jets in DJANGO is accounted for using the colourdipole model [30]. The contribution to the SM background from lepton pair production $e p \rightarrow e \ell^{+} \ell^{-} X$ is calculated using the GRAPE [31] generator, based on a full set of electroweak diagrams. The production mechanisms include $\gamma \gamma$, $\gamma Z, Z Z$ interactions, internal photon conversion and the decay of virtual or real $Z$ bosons.

Generated events are passed through the full GEANT [32] based simulation of the $\mathrm{H} 1$ detector, which takes into account the running conditions of the different data taking periods, and are reconstructed and analysed using the same program chain as for the data.

\section{Experimental conditions}

The data were recorded at electron and proton beam energies of $27.6 \mathrm{GeV}$ and $820 \mathrm{GeV}$ or $920 \mathrm{GeV}$, corresponding to centre-of-mass energies $\sqrt{s}$ of $301 \mathrm{GeV}$ or $319 \mathrm{GeV}$, respectively. The total integrated luminosity of the analysed data is $474 \mathrm{pb}^{-1}$, which represents a factor of four increase with respect to the previous published results. The data set is made up of $183 \mathrm{pb}^{-1}$ recorded in $e^{-} p$ collisions (1998-2006) and $291 \mathrm{pb}^{-1}$ in $e^{+} p$ collisions (1994-2007), of which $36 \mathrm{pb}^{-1}$ were recorded at $\sqrt{s}=301 \mathrm{GeV}$ (19941997). Data collected from 2003 onwards were taken with a longitudinally polarised lepton beam, with polarisation typically at a level of $35 \%$. The residual polarisation of the combined left-handed and right-handed data periods is about $2 \%$ left-handed. While previous measurements were performed using mainly $e^{+} p$ data, an $e^{-} p$ data set with more than a ten-fold increase in integrated luminosity is now analysed.

A detailed description of the $\mathrm{H} 1$ experiment can be found in $[33,34]$. Only the detector components relevant to the present analysis are briefly described here. The origin of the H1 coordinate system is the nominal ep interaction point, with the direction of the proton beam defining the positive $z$-axis (forward region). Transverse momentum is measured in the $x-y$ plane. The pseudorapidity $\eta$ is related to the polar angle $\theta$ by $\eta=-\ln \tan (\theta / 2)$.

The Liquid Argon (LAr) calorimeter [35] covers the polar angle range $4^{\circ}<\theta<154^{\circ}$ with full azimuthal acceptance. Electromagnetic shower energies are measured with a 
precision of $\sigma(E) / E=12 \% / \sqrt{E / \mathrm{GeV}} \oplus 1 \%$ and hadronic energies with $\sigma(E) / E=50 \% / \sqrt{E / \mathrm{GeV}} \oplus 2 \%$, as measured in test beams $[36,37]$. In the backward region, energy measurements are provided by a lead/scintillating-fibre calorimeter ${ }^{2}$ (SpaCal) [38] covering the range $155^{\circ}<\theta<$ $178^{\circ}$.

The central $\left(20^{\circ}<\theta<160^{\circ}\right)$ and forward $\left(7^{\circ}<\theta<\right.$ $25^{\circ}$ ) inner tracking detectors are used to measure charged particle trajectories, to reconstruct the interaction vertex and to complement the measurement of hadronic energies. In each event the tracks are used in a common fit procedure to determine the $e p$ interaction vertex. Tracks not fitted to the event vertex are referred to as NV tracks in the following. The LAr calorimeter and inner tracking detectors are enclosed in a super-conducting magnetic coil with a field strength of $1.16 \mathrm{~T}$. From the curvature of charged particle trajectories in the magnetic field, the central tracking system provides transverse momentum measurements with a resolution of $\sigma_{P_{T}} / P_{T}=0.005 P_{T} / \mathrm{GeV} \oplus 0.015$ [39]. The return yoke of the magnetic coil is the outermost part of the detector and is equipped with streamer tubes forming the central muon detector and tail catcher $\left(4^{\circ}<\theta<171^{\circ}\right)$. In the forward region $\left(3^{\circ}<\theta<17^{\circ}\right)$ a set of drift chamber layers forms the forward muon detector, which together with an iron toroidal magnet allows a momentum measurement.

The luminosity is determined from the rate of the BetheHeitler process $e p \rightarrow e p \gamma$, measured using a photon detector located close to the beam pipe at $z=-103 \mathrm{~m}$, in the backward direction.

The LAr calorimeter provides the main trigger for events in this analysis [40]. The trigger efficiency is almost $100 \%$ for events containing an electron with a transverse momentum above $10 \mathrm{GeV}$. For events with a transverse momentum imbalance of $12 \mathrm{GeV}$ measured in the calorimeter the trigger efficiency is about $60 \%$, rising to about $98 \%$ for an imbalance greater than $25 \mathrm{GeV}$. In addition to the calorimeter trigger, muon events may also be triggered by a pattern consistent with a minimum ionising particle in the muon system in coincidence with tracks in the tracking detectors.

In order to remove events induced by cosmic rays and other non-ep background, the event vertex is required to be reconstructed within $\pm 35 \mathrm{~cm}$ in $z$ of the average nominal interaction point. In addition, topological filters and timing vetoes are applied.

\section{Particle identification and event kinematics}

The identification of electrons is based on the measurement [41] of a compact and isolated electromagnetic cluster in the LAr calorimeter or SpaCal. The energy measured

\footnotetext{
${ }^{2}$ This device was installed in 1995, replacing a lead-scintillator sandwich calorimeter [33, 34].
}

within a cone in pseudorapidity-azimuth $(\eta-\phi)$ of radius $R=\sqrt{\Delta \eta^{2}+\Delta \phi^{2}}=0.5$ around the electron is required to be less than $3 \%$ of the total cluster energy. For $\theta_{e}>20^{\circ}$, electrons are also required to have an associated track with an extrapolated distance of closest approach (DCA) to the cluster of less than $12 \mathrm{~cm}$. The distance from the first measured track point in the central drift chambers to the beam axis is required to be below $30 \mathrm{~cm}$ in order to reject photons that convert late in the central tracker material. For $\theta_{e}<20^{\circ}$, no track conditions are imposed, but an additional calorimetric isolation criterion is used, where the energy in a cone of radius $R=0.75$ is required to be less than $5 \%$ of the total cluster energy. The charge of the electron, measured from the associated track, is used for events with $\theta_{e}>20^{\circ}$ and if the charge is measured with a significance $\sigma_{q}=|\kappa| / \delta \kappa>1.0$, where the curvature $|\kappa|$ of the track is proportional to $1 / P_{T}^{\text {track }}$ and $\delta \kappa$ is the associated error on the curvature measurement. Electrons found in regions between calorimeter modules containing large amounts of inactive material are excluded [42]. The energy and polar angle of the electron are measured from the calorimeter cluster. The azimuthal angle is taken from the associated track. The calibration of the electromagnetic part of the LAr calorimeter is performed using NC events with the method described in [42].

Muon identification is based on the measurement of a track in the inner tracking systems associated with a track or an energy deposit in the central muon detector or forward muon detector $[43,44]$. Muons which do not reach the muon detector, or enter inefficient regions of the muon detector, may be identified by a central track linked to a signature of a minimal ionising particle in the LAr calorimeter and a hit in the tail catcher. The muon momentum is measured from the track curvature in the solenoidal or toroidal magnetic field. For very high energy muons, the curvature may be compatible with zero within two standard deviations of its error $\delta \kappa$, and in such cases is re-evaluated at $|\kappa|+\delta \kappa$, allowing a lower limit to be placed on the transverse momentum. A muon may have no more than $5 \mathrm{GeV}$ deposited in a cylinder, centred on the muon track direction, of radius $25 \mathrm{~cm}$ and $50 \mathrm{~cm}$ in the electromagnetic and hadronic sections of the LAr calorimeter, respectively.

Calorimeter energy deposits and tracks not previously identified as electron or muons are used to form combined cluster-track objects, from which the hadronic final state is reconstructed $[45,46]$. Jets with a minimum transverse momentum of $4 \mathrm{GeV}$ are reconstructed from these combined cluster-track objects using an inclusive $k_{T}$ algorithm [47, 48].

The isolation of leptons with respect to jets and tracks in the event is quantified using the distances of separation in $(\eta-\phi)$ space $D(\ell ;$ jet $)$ and $D(\ell$; track $)$, respectively.

Hadronic one-prong decays of tau leptons are identified using isolated narrow jets with a track multiplicity of one. 
Such tau candidate jets are only identified in the dedicated search described in Sect. 5.2.

The following kinematic quantities are employed in the analysis, some of which are sensitive to the presence of undetected high energy particles and/or can be used to reduce the main background contributions:

- $P_{T}^{X}$, the transverse momentum of the inclusive hadronic final state.

- $P_{T}^{\text {miss }}$, the total missing transverse momentum calculated from all reconstructed particles. In events with large $P_{T}^{\text {miss }}$, the only non-detected particle in the event is assumed to be a neutrino.

- $P_{T}^{\text {calo }}$, the net transverse momentum calculated from all reconstructed particles, where for muons only the energy deposited in the calorimeter is included. For events containing high energy muons $P_{T}^{\text {calo }} \simeq P_{T}^{X}$ (the hadronic transverse momentum), otherwise $P_{T}^{\text {calo }}=P_{T}^{\text {miss }}$.

- $V_{\mathrm{ap}} / V_{\mathrm{p}}$, a measure of the azimuthal balance of the event. It is defined as the ratio of the anti-parallel to parallel components of all measured calorimetric clusters, with respect to the direction of the calorimetric transverse momentum [42].

- $\Delta \phi_{\ell-X}$, the difference in azimuthal angle between the lepton and the direction of $P_{T}^{X}$. For events with low hadronic transverse momentum $P_{T}^{X}<1.0 \mathrm{GeV}, \Delta \phi_{\ell-X}$ is set to zero.

- $\delta_{\text {miss }}=2 E_{e}^{0}-\sum_{i}\left(E^{i}-P_{z}^{i}\right)$, where the sum runs over all detected particles, $P_{z}$ is the momentum along the proton beam axis and $E_{e}^{0}$ is the electron beam energy. For an event where only momentum in the proton direction is undetected, $\delta_{\text {miss }}$ is zero.

- $\zeta_{e}^{2}=4 E_{e} E_{e}^{0} \cos ^{2} \theta_{e} / 2$, where $E_{e}$ is the energy of the final state electron. For NC events, where the scattered elec- tron is identified as the isolated high transverse momentum electron, $\zeta_{e}^{2}$ is equal to the four momentum transfer squared $Q_{e}^{2}$, as measured by the electron method [49].

\section{Event selection}

\subsection{Events with isolated electrons and muons}

The event selection for isolated electrons and muons is based on the analysis described in $[3,50,51]$ and is summarised in Table 1 . The lepton is required to have a high transverse momentum $P_{T}^{\ell}>10 \mathrm{GeV}$, be observed in the central or forward region of the detector, $5^{\circ}<\theta_{\ell}<140^{\circ}$, and be isolated with respect to jets and other tracks in the event. A large transverse momentum imbalance $P_{T}^{\text {miss }}$ is required and the cut on $P_{T}^{\text {calo }}$ is imposed to ensure a high trigger efficiency.

In order to reject the NC background contribution in the electron channel, further cuts are applied on the longitudinal momentum imbalance $\delta_{\text {miss }}$ and the azimuthal balance of the event, using $V_{\mathrm{ap}} / V_{\mathrm{p}}$ and $\Delta \phi_{e-X}$. The cut on $\delta_{\text {miss }}$ is only performed if the event contains exactly one electron, which has the same charge as the beam lepton. Electron events with low values of $P_{T}^{\text {calo }}$ are additionally required to have large $\zeta_{e}^{2}$ in order to further reduce NC background, which is predominantly at low $Q_{e}^{2}$. Lepton pair background is removed from the muon channel by rejecting azimuthally balanced events, using $V_{\text {ap }} / V_{\mathrm{p}}$ and $\Delta \phi_{\mu-X}$, and by rejecting events with two or more isolated muons. To ensure that the two lepton channels are exclusive, electron events must contain no isolated muons.

Some changes have been made to the selection with respect to that used in the previous publication [3]. The cut
Table 1 Summary of selection requirements for the electron and muon channels in the search for events with isolated leptons and missing transverse momentum

\begin{tabular}{|c|c|c|c|}
\hline \multicolumn{4}{|c|}{$\mathrm{H} 1$ isolated lepton $+P_{T}^{\text {miss }}$ event selection } \\
\hline & Electron & & Muon \\
\hline $\begin{array}{l}\text { Basic event } \\
\text { selection }\end{array}$ & & $\begin{array}{l}5^{\circ}<\theta_{\ell}<140^{\circ} \\
P_{T}^{\ell}>10 \mathrm{GeV} \\
P_{T}^{\text {miss }}>12 \mathrm{GeV} \\
P_{T}^{\text {calo }}>12 \mathrm{GeV}\end{array}$ & \\
\hline \multirow[t]{2}{*}{ Lepton isolation } & & $D(\ell ;$ jet $)>1.0$ & \\
\hline & $D(e ;$ track $)>0.5$ for $\theta_{e}>45^{\circ}$ & & $D(\mu ;$ track $)>0.5$ \\
\hline \multirow{7}{*}{$\begin{array}{l}\text { Background } \\
\text { rejection }\end{array}$} & & $V_{\text {ap }} / V_{\mathrm{p}}<0.5$ & \\
\hline & $V_{\mathrm{ap}} / V_{\mathrm{p}}<0.2$ for $P_{T}^{e}<25 \mathrm{GeV}$ & & $V_{\text {ap }} / V_{\mathrm{p}}<0.2$ for $P_{T}^{\text {calo }}<25 \mathrm{GeV}$ \\
\hline & $\Delta \phi_{e-X}<160^{\circ}$ & & $\Delta \phi_{\mu-X}<170^{\circ}$ \\
\hline & $\delta_{\text {miss }}>5 \mathrm{GeV}$ & & - \\
\hline & $\zeta_{e}^{2}>5000 \mathrm{GeV}^{2}$ for $P_{T}^{\text {calo }}<25 \mathrm{GeV}$ & & - \\
\hline & & $M_{T}^{\ell \nu}>10 \mathrm{GeV}$ & \\
\hline & - & & $P_{T}^{X}>12 \mathrm{GeV}$ \\
\hline
\end{tabular}


on $V_{\mathrm{ap}} / V_{\mathrm{p}}$ at low transverse momentum in both the electron and muon channels has been relaxed to 0.2 , leading to an improved signal efficiency. As described in Sect. 4, in the very forward region $\theta_{e}<20^{\circ}$ the electron DCA requirement is replaced by a stricter calorimetric isolation criterion, which results in a better CC rejection. Finally, the lepton-neutrino transverse mass:

$M_{T}^{\ell v}=\sqrt{\left(P_{T}^{\text {miss }}+P_{T}^{\ell}\right)^{2}-\left(\vec{P}_{T}^{\text {miss }}+\vec{P}_{T}^{\ell}\right)^{2}}$

is required to be larger than $10 \mathrm{GeV}$ in order to further reject NC (lepton pair) background in the electron (muon) channel.

Following the selection criteria described above, the overall efficiency to select SM $W \rightarrow e v$ events is $44 \%$ and to select $\mathrm{SM} W \rightarrow \mu \nu$ events is $17 \%$, calculated using the EPVEC simulation. The main difference in efficiency between the two channels is due to the cut on $P_{T}^{\text {calo }}$, which for muon events effectively acts as a cut on $P_{T}^{X}$. As the efficiency for muons at low $P_{T}^{X}$ is small, an additional requirement is made of $P_{T}^{X}>12 \mathrm{GeV}$. For events with $P_{T}^{X}>25 \mathrm{GeV}$ the selection efficiencies of the two channels are comparable at $\sim 42 \%$.

\subsection{Events with isolated tau leptons}

The selection of events with isolated tau leptons and missing transverse momentum is based on the analysis described in [14] and is summarised in Table 2. The tau identification algorithm selects narrow (pencil-like), low multiplicity jets typical for hadronic tau decays. Only hadronic decays with one charged hadron (one-prong) are considered. Tau decays to electrons and muons enter the electron and muon channels described in Sect. 5.1.

To ensure the presence of neutrinos in the event, large $P_{T}^{\text {miss }}$ and $P_{T}^{\text {calo }}$, significant $\delta_{\text {miss }}$ and low $V_{\mathrm{ap}} / V_{\mathrm{p}}$ are required. The event should also exhibit large inclusive hadronic transverse momentum $P_{T}^{X}$. At this stage the selection contains $96 \% \mathrm{CC}$ events and is denoted CC-like in the following.

Tau jet candidates are based on jets found in the hadronic final state. The tau lepton four-vector is approximated using the four-vector of the tau jet candidate. The tau lepton four-vector is subtracted from the inclusive hadronic final state $X$ to obtain the transverse momentum of the remaining hadronic system $X^{\prime}$ as:

$\vec{P}_{T}^{X^{\prime}}=\vec{P}_{T}^{X}-\vec{P}_{T}^{\tau}$

Jets with transverse momentum $P_{T}^{\text {jet }}>7 \mathrm{GeV}$ found in the central region $20^{\circ}<\theta_{\text {jet }}<120^{\circ}$ of the detector are considered as tau jet candidates. The angular region is reduced with respect to that used in the electron and muon channels since in the forward region the track multiplicity cannot be reliably measured and in the backward region the rate from
Table 2 Summary of selection requirements for isolated tau jets in events with missing transverse momentum. $N_{\text {tracks }}^{D \text { jet }}{ }^{1.0}$ denotes the number of tracks in a cone of 1.0 in the $(\eta-\phi)$ plane around the jet-axis. $N_{\mathrm{NV} \text { tracks }}^{D \text { trac }<0.3}$ denotes the number of NV tracks in a cone of 0.3 in the $(\eta-\phi)$ plane around the one-prong track

$\mathrm{H} 1$ isolated tau lepton $+P_{T}^{\text {miss }}$ event selection

\begin{tabular}{ll}
\hline CC-like sample & $P_{T}^{\text {miss }}>12 \mathrm{GeV}$ \\
& $P_{T}^{\text {calo }}>12 \mathrm{GeV}$ \\
& $P_{T}^{X}>12 \mathrm{GeV}$ \\
& $\delta_{\text {miss }}>5 \mathrm{GeV}$ \\
& $V_{\text {ap }} / V_{\mathrm{p}}<0.5$ \\
& $V_{\text {ap }} / V_{\mathrm{p}}<0.15$ for $P_{T}^{\text {miss }}<25 \mathrm{GeV}$ \\
& $P_{T}^{\text {jet }}>7 \mathrm{GeV}$ \\
& $20^{\circ}<\theta_{\text {jet }}<120^{\circ}$ \\
Tau-like jets & $R_{\text {jet }}<0.12$ \\
& $N_{\text {tracks }}^{\text {jet }} \geq 1$ for $P_{T}^{\text {track }}>5 \mathrm{GeV}$ \\
& $D(\tau ; e, \mu$, jet $)>1.0$ \\
Isolation & $\Delta \phi_{\tau-X^{\prime}}<170^{\circ}$ for $P_{T}^{X^{\prime}}>5 \mathrm{GeV}$ \\
Acoplanarity & $N_{\text {tracks }}^{D \text { jet }<1.0}=1$ \\
One-prong & $N_{\mathrm{NV} \text { tracks }}^{D \text { track }}=1$ \\
\hline
\end{tabular}

NC DIS is high, leading to a correspondingly high expectation of narrow jets from falsely identified electrons. The jet radius is used as a measure for the collimation of the jet and is calculated as:

$R_{\text {jet }}=\frac{1}{E_{\text {jet }}} \sum_{h} E_{h} \sqrt{\Delta \eta(\text { jet }, h)^{2}+\Delta \phi(\text { jet }, h)^{2}}$,

where $E_{\text {jet }}$ is the total jet energy and the sum runs over all jet daughter hadronic final state particles of energy $E_{h}$. Narrow jets are selected by requiring $R_{\text {jet }}<0.12$. At least one track measured in the central tracking detector with transverse momentum $P_{T}^{\text {track }}>5 \mathrm{GeV}$ is required to be associated to the jet. Jets meeting the above criteria are denoted tau-like jets in the following and are investigated in control samples as described in Appendix A.3.

For the final selection, the tau jet should be isolated from electrons, muons and other jets in the event by requiring a minimum separation $D(\tau ; e, \mu$, jet $)>1.0$. To avoid the misidentification of jets or electrons as tau jets due to distortion of the shower shapes, jets pointing to regions between calorimeter modules containing large amounts of inactive material are not considered [42]. Acoplanarity between the tau jet and the remaining hadronic system $X^{\prime}$ in the transverse plane $\Delta \phi_{\tau-X^{\prime}}$ is required to suppress events with back-to-back topologies, primarily NC events and photoproduction events with jets. Finally, a track multiplicity of one is required in a cone of radius $R=1.0$ around the jet axis. To improve the rejection of low multiplicity hadronic 
jets with additional charged hadrons, no further NV tracks are allowed in a cone of radius $R=0.3$ around the oneprong track.

\section{Systematic uncertainties}

The following experimental systematic uncertainties are considered:

- The uncertainty on the electromagnetic energy scale varies depending on the polar angle from $0.7 \%$ in the backward region up to $2 \%$ in the forward region. The uncertainties on the $\theta_{e}$ and $\phi_{e}$ measurements are $3 \mathrm{mrad}$ and $1 \mathrm{mrad}$, respectively. The identification efficiency of electrons is known with an uncertainty of $2 \%$.

- The scale uncertainty on the transverse momentum of high $P_{T}$ muons is $2.5 \%$. The uncertainties on the $\theta_{\mu}$ and $\phi_{\mu}$ measurements are $3 \mathrm{mrad}$ and $1 \mathrm{mrad}$, respectively. The muon identification efficiency has an error of $5 \%$ in the region $\theta_{\mu}>12.5^{\circ}$ and $15 \%$ in the region $\theta_{\mu}<12.5^{\circ}$.

- The hadronic energy scale is known within $2 \%$ for events with $P_{T}^{X}>8 \mathrm{GeV}$ and $5 \%$ for events with $P_{T}^{X}<8 \mathrm{GeV}$. The uncertainties on the $\theta$ and $\phi$ measurements of the hadronic final state are both $10 \mathrm{mrad}$. The error on the measurement of $V_{\text {ap }} / V_{\mathrm{p}}$ is \pm 0.02 .

- The uncertainty on the track reconstruction efficiency is $3 \%$.

- The uncertainty on the trigger efficiency for the muon channel varies from $3.4 \%$ for events with $P_{T}^{X}>12 \mathrm{GeV}$ to $1.3 \%$ for events with $P_{T}^{X}>25 \mathrm{GeV}$.

- The luminosity measurement has an uncertainty of $3 \%$.

The effect of the above systematic uncertainties on the SM expectation is determined by varying the experimental quantities by \pm 1 standard deviation in the MC samples and propagating these variations through the whole analysis. The contribution to the total error in the combined electron and muon channels from the experimental systematic uncertainties is $4.5 \%$ at low $P_{T}^{X}$, rising to $8.1 \%$ for events with $P_{T}^{X}>25 \mathrm{GeV}$.

A theoretical uncertainty of $15 \%$ is quoted for the predicted contributions from signal processes. This is mainly due to uncertainties in the parton distribution functions and the scales at which the calculation is performed [17-19].

Additional model systematic uncertainties are attributed to the SM background MC generators described in Sect. 2, deduced from the level of agreement observed between the data and simulations in dedicated samples, as described in Appendix A.

In the electron and muon channels, the contributions from background processes modelled using RAPGAP (NC), PYTHIA (photoproduction), GRAPE (lepton pair) and WABGEN (QED Compton) are attributed an error of 30\%, and the $\mathrm{CC}$ background contribution, which is modelled using DJANGO, is attributed an error of 50\%. The uncertainties associated with lepton misidentification and the measurement of missing transverse momentum, as well as the normalisation of NC DIS and photoproduction processes with at least two high $P_{T}$ jets, are included in these errors.

In the tau channel, the contributions from background processes modelled using RAPGAP are attributed a 15\% model error and from PYTHIA a $20 \%$ model error. The DJANGO prediction is attributed an error of $20 \%$ and the GRAPE prediction is attributed an error of $30 \%$. In addition to these model uncertainties, an uncertainty on the description of the jet radius is considered in the tau channel. The jet radius is varied by \pm 0.012 , corresponding to $10 \%$ of the cut value, resulting in a $17 \%$ change in the rate of tau-like jets selected in the CC-like sample. All other experimental systematic uncertainties in the tau channel are included in the model uncertainties.

In the combination of the electron and muon channels all systematic errors are treated as fully correlated. The total error on the SM prediction is determined by adding the MC statistical error to the effects of all model and experimental systematic uncertainties in quadrature.

\section{Results}

\subsection{Electron and muon channels}

The results of the search for events with an isolated electron or muon and missing transverse momentum are summarised in Table 3. In the full $e^{ \pm} p$ data sample 53 events are observed, in good agreement with the SM prediction of $54.1 \pm 7.4$, where $40.4 \pm 6.3$ events are predicted from signal processes, dominated by single $W$ production. In the $e^{+} p$ data 40 events are observed compared to a SM prediction of $32.3 \pm 4.4$. In the $e^{-} p$ data 13 events are observed compared to a SM prediction of $21.8 \pm 3.1$. Two of the events in the electron channel observed in the previous analysis [3] are rejected in the present analysis due to the tighter calorimetric isolation requirement described in Sect. 4.

In the complete data set, 39 electron events are observed, compared to a SM prediction of $43.1 \pm 6.0$. In 17 of the electron events the lepton charge is measured as positive, in 9 events negative and is unmeasured in the remaining 13 events. Similarly for the muon channel, 14 data events are observed, compared to a SM prediction of $11.0 \pm 1.8$. In 5 of the muon events the lepton charge is measured as positive, in 6 events negative and is unmeasured in the remaining 3 events.

Kinematic distributions of the selected data events are shown in Fig. 2. The events are observed to have generally 
Table 3 Summary of the H1 results of searches for events with isolated electrons or muons and missing transverse momentum for the $e^{+} p$ data (291 $\mathrm{pb}^{-1}$, top), $e^{-} p$ data $\left(183 \mathrm{pb}^{-1}\right.$, middle) and the full HERA data set $\left(474 \mathrm{pb}^{-1}\right.$, bottom). The results are shown for the full selected sample and for the subsample at large transverse momentum $P_{T}^{X}>25 \mathrm{GeV}$. The number of observed events is compared to the SM prediction. The SM signal (dominated by single $W$ production) and the total background contribution are also shown. The quoted errors contain statistical and systematic uncertainties added in quadrature

\begin{tabular}{|c|c|c|c|c|c|}
\hline $\mathrm{H} 1$ & $1994-2007 e^{+} p 291 \mathrm{pb}^{-1}$ & Data & SM expectation & SM signal & Other SM processes \\
\hline \multirow[t]{2}{*}{ Electron } & Total & 28 & $25.6 \pm 3.5$ & $18.6 \pm 2.9$ & $6.9 \pm 1.7$ \\
\hline & $P_{T}^{X}>25 \mathrm{GeV}$ & 9 & $4.32 \pm 0.71$ & $3.56 \pm 0.61$ & $0.76 \pm 0.32$ \\
\hline \multirow[t]{2}{*}{ Muon } & Total & 12 & $6.7 \pm 1.1$ & $6.1 \pm 1.0$ & $0.55 \pm 0.18$ \\
\hline & $P_{T}^{X}>25 \mathrm{GeV}$ & 8 & $3.70 \pm 0.63$ & $3.43 \pm 0.60$ & $0.28 \pm 0.09$ \\
\hline \multirow[t]{2}{*}{ Combined } & Total & 40 & $32.3 \pm 4.4$ & $24.8 \pm 3.9$ & $7.5 \pm 1.8$ \\
\hline & $P_{T}^{X}>25 \mathrm{GeV}$ & 17 & $8.0 \pm 1.3$ & $7.0 \pm 1.2$ & $1.04 \pm 0.37$ \\
\hline $\mathrm{H} 1$ & $1998-2006 e^{-} p 183 \mathrm{pb}^{-1}$ & Data & SM expectation & SM signal & Other SM processes \\
\hline \multirow[t]{2}{*}{ Electron } & Total & 11 & $17.5 \pm 2.7$ & $11.6 \pm 1.8$ & $5.9 \pm 1.9$ \\
\hline & $P_{T}^{X}>25 \mathrm{GeV}$ & 1 & $3.18 \pm 0.59$ & $2.23 \pm 0.38$ & $0.95 \pm 0.41$ \\
\hline \multirow[t]{2}{*}{ Muon } & Total & 2 & $4.29 \pm 0.69$ & $3.96 \pm 0.66$ & $0.33 \pm 0.11$ \\
\hline & $P_{T}^{X}>25 \mathrm{GeV}$ & 0 & $2.40 \pm 0.41$ & $2.22 \pm 0.39$ & $0.19 \pm 0.06$ \\
\hline \multirow[t]{2}{*}{ Combined } & Total & 13 & $21.8 \pm 3.1$ & $15.6 \pm 2.4$ & $6.2 \pm 1.9$ \\
\hline & $P_{T}^{X}>25 \mathrm{GeV}$ & 1 & $5.58 \pm 0.91$ & $4.45 \pm 0.75$ & $1.14 \pm 0.44$ \\
\hline $\mathrm{H} 1$ & $1994-2007 e^{ \pm} p 474 \mathrm{pb}^{-1}$ & Data & SM expectation & SM signal & Other SM processes \\
\hline \multirow[t]{2}{*}{ Electron } & Total & 39 & $43.1 \pm 6.0$ & $30.3 \pm 4.8$ & $12.8 \pm 3.4$ \\
\hline & $P_{T}^{X}>25 \mathrm{GeV}$ & 10 & $7.5 \pm 1.3$ & $5.79 \pm 0.99$ & $1.71 \pm 0.72$ \\
\hline \multirow[t]{2}{*}{ Muon } & Total & 14 & $11.0 \pm 1.8$ & $10.1 \pm 1.7$ & $0.88 \pm 0.28$ \\
\hline & $P_{T}^{X}>25 \mathrm{GeV}$ & 8 & $6.1 \pm 1.0$ & $5.64 \pm 0.99$ & $0.47 \pm 0.15$ \\
\hline \multirow[t]{2}{*}{ Combined } & Total & 53 & $54.1 \pm 7.4$ & $40.4 \pm 6.3$ & $13.7 \pm 3.5$ \\
\hline & $P_{T}^{X}>25 \mathrm{GeV}$ & 18 & $13.6 \pm 2.2$ & $11.4 \pm 1.9$ & $2.18 \pm 0.80$ \\
\hline
\end{tabular}

low values of lepton polar angle and have a $\Delta \phi_{\ell-X}$ distribution in agreement with the SM prediction. The shape of the transverse mass $M_{T}^{\ell v}$ distribution shows a Jacobian peak as expected from single $W$ production. The observed $P_{T}^{X}$, $P_{T}^{\text {miss }}$ and $P_{T}^{\ell}$ distributions are also indicative of $W$ production, where the decay products of the $W$ peak around $40 \mathrm{GeV}$ and the hadronic final state has typically low $P_{T}^{X}$. In 11 of the 53 events in the final sample an additional electron is observed in the main detector, in agreement with a SM prediction of $11.7 \pm 1.5$. In $W$ production events the additional electron typically corresponds to the scattered electron, as illustrated in Fig. 1(a).

Figure 3 displays some examples of data events selected by the analysis. Event (a) features a high $P_{T}$ electron, large missing transverse momentum and no visible reconstructed hadronic final state, which is typical of low $P_{T}$ single $W$ production. Event (b) features a high $P_{T}$ muon, in addition to a prominent hadronic jet, resulting in a large $P_{T}^{X}$. The presence of an undetected particle in the event is particularly visible in the transverse $(x-y)$ plane.

In the region $P_{T}^{X}>25 \mathrm{GeV}, 18$ events are observed compared to an expectation of $13.6 \pm 2.2$, as shown in Table 3 .
Almost all of the high $P_{T}^{X}$ events are seen in the $e^{+} p$ data, where 17 events are observed compared to an expectation of $8.0 \pm 1.3$. This excess of data events over the SM prediction corresponds to a $2.4 \sigma$ significance, which is lower than previously reported in [3]. In the $e^{-} p$ data no excess is seen, where only 1 event is observed in the data with $P_{T}^{X}>25 \mathrm{GeV}$, compared to a SM expectation of $5.6 \pm 0.9$. The kinematics of the events with $P_{T}^{X}>25 \mathrm{GeV}$ are detailed in Table 4.

The hadronic transverse momentum distribution is shown separately for $e^{+} p$ and $e^{-} p$ data in Fig. 4. In addition to the slight excess of $e^{+} p$ data events visible at high $P_{T}^{X}$, a slight deficit of data events is visible in the $e^{-} p$ sample, particularly at low values of $P_{T}^{X}$. Potential sources of inefficiency in the data due to data quality requirements, such as trigger conditions, the vertex requirement and non-ep background suppression, were investigated and ruled out as the origin of the observed differences between the $e^{+} p$ and $e^{-} p$ results. A series of control samples, enriched with SM background physics processes, are also used to investigate these observations in the final sample, as discussed in Appen$\operatorname{dix} \mathrm{A}$. 
Fig. 2 Distributions in the combined electron and muon channels for the $e^{ \pm} p$ data sample. Shown is the polar angle of the lepton $\theta_{\ell}(\mathbf{a})$, the lepton-hadronic system acoplanarity $\Delta \phi_{\ell-X}(\mathbf{b})$, the lepton-neutrino transverse mass $M_{T}^{\ell v}(\mathbf{c})$, the hadronic transverse momentum $P_{T}^{X}(\mathbf{d})$, the missing transverse momentum $P_{T}^{\text {miss }}$ (e) and the transverse momentum of the lepton $P_{T}^{\ell}(\mathbf{f})$. The data (points) are compared to the SM expectation (open histogram). The signal component of the SM expectation, dominated by real $W$ production, is shown as the hatched histogram. The total uncertainty on the SM expectation is shown as the shaded band

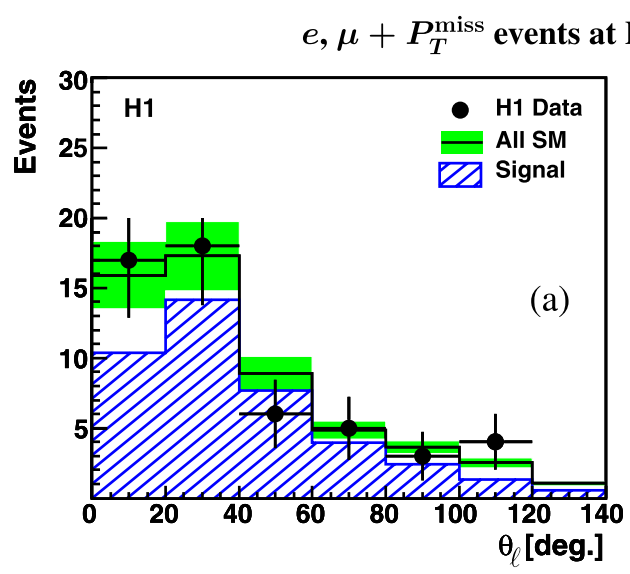

HERA $\left(e^{ \pm} p, 474 \mathrm{pb}^{-1}\right)$
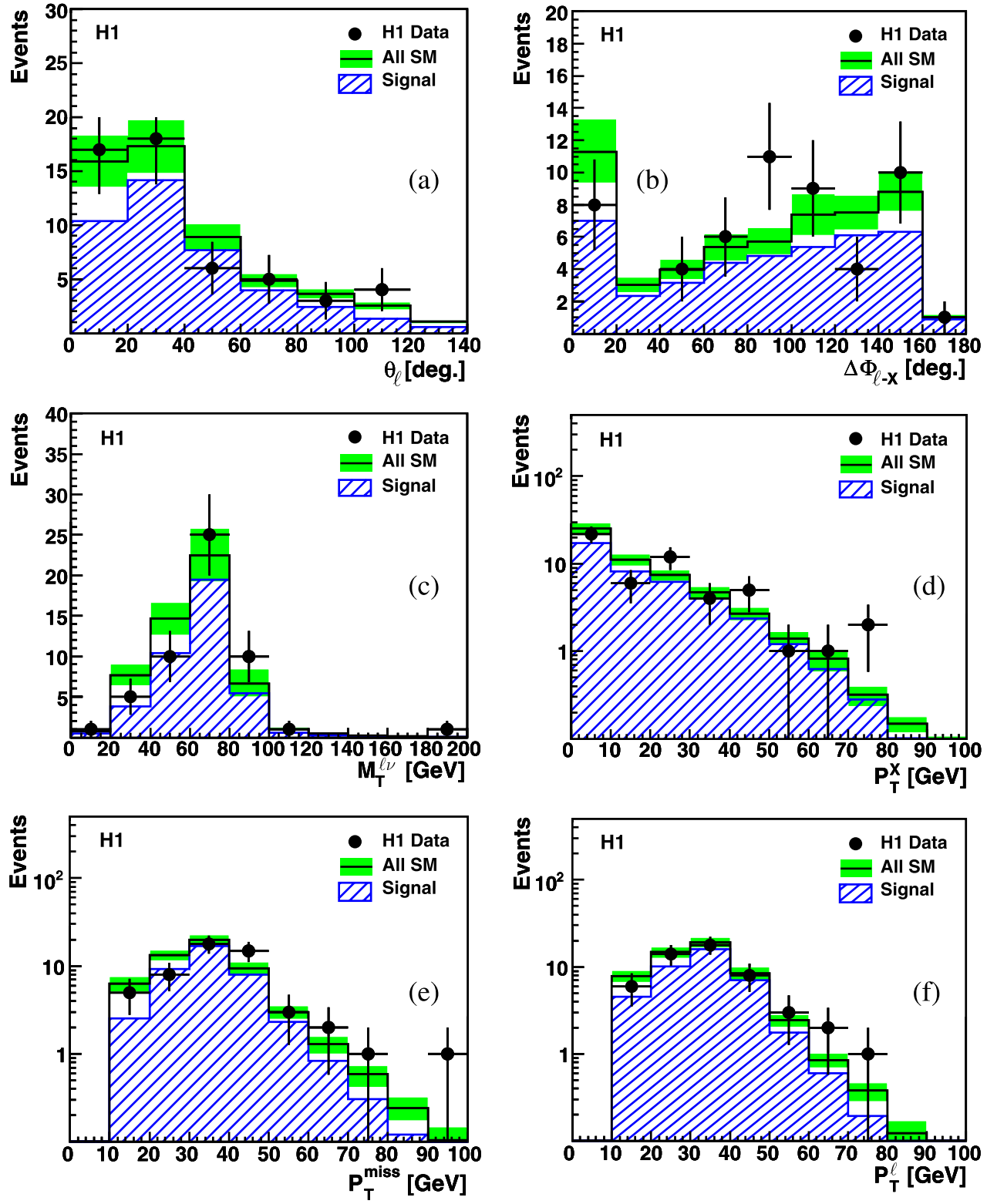

\subsection{Tau lepton channel}

The results of the search in the tau channel are summarised in Table 5. In the final event sample, 18 events are selected, compared to a SM expectation of $23.2 \pm 3.8$. The SM expectation is dominated by charged current background processes, whereas the signal contribution is only $11 \%$. Distributions of the events in the final sample are shown in Fig. 5. Most of the events are observed at very low $P_{T}^{X^{\prime}}$. At $P_{T}^{X^{\prime}}>25 \mathrm{GeV}$ one event is observed in the data, compared to a SM expectation of $1.5 \pm 0.2$. In this region the contribution of single $W$ boson production to the SM expectation is about 38\%. The data event, shown in Fig. 3(c), is selected in $e^{-} p$ collisions and exhibits $P_{T}^{\tau}=14.3 \pm 1.2 \mathrm{GeV}$,
$P_{T}^{X^{\prime}}=62 \pm 5 \mathrm{GeV}$ and $P_{T}^{\text {miss }}=68 \pm 6 \mathrm{GeV}$. Of the six events observed in the previous analysis [14], three are retained in this selection, whereas the other three are rejected by the tighter track isolation described in Sect. 5.2.

The low efficiency to detect tau-like jets of about $16 \%$, together with the branching ratio to hadronic one-prong decays of $49 \%$ and the restricted polar angular range, leads to a significantly lower SM expectation of $W \rightarrow \tau v_{\tau}$ events of only $2.7 \pm 0.4$ events, compared to $30.3 \pm 4.8$ and $10.1 \pm 1.7$ in the electron and muon channels, respectively. A comparison and cross check of the electron and muon channel is impeded by this low efficiency and the high background contamination, and therefore the tau channel is not included in the cross section measurements described below. 
Fig. 3 Displays of isolated lepton events in the final sample. (a) An electron event with missing transverse momentum and no visible hadronic final state, typical of low $P_{T}$ single $W$ production. (b) An isolated muon event, with missing transverse momentum and a prominent hadronic final state $X$.

(c) An event with an isolated tau lepton candidate, missing transverse momentum and a prominent hadronic final state $X$

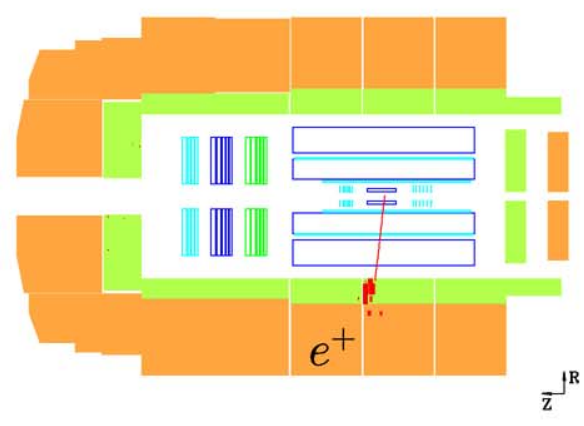

(a) Run 364631 Event 64013

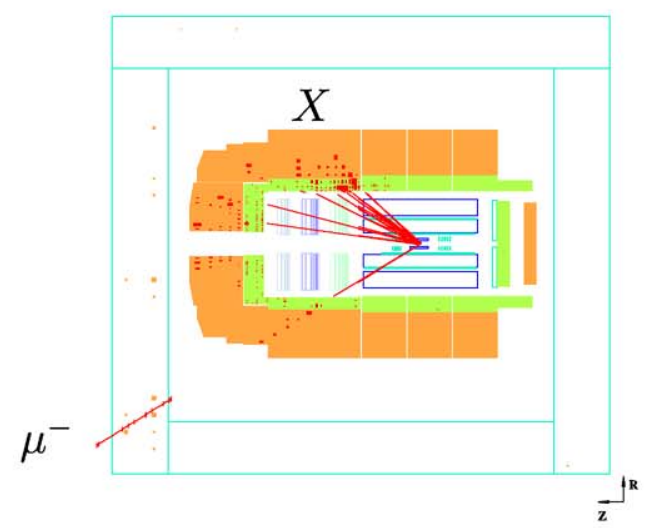

(b) Run 473929 Event 107593

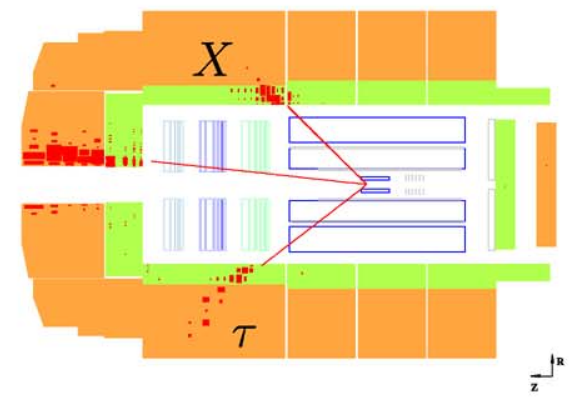

(c) Run 417955 Event 105857

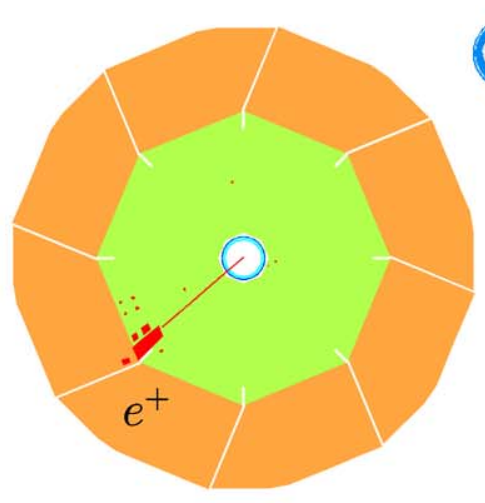

$\vec{X}^{1 Y}$

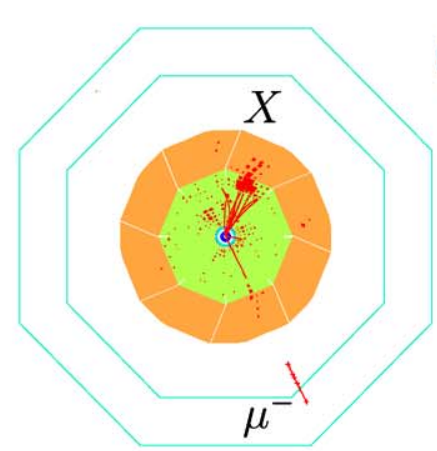

$\vec{x}^{y x}$$$
\overrightarrow{\mathrm{x}}
$$
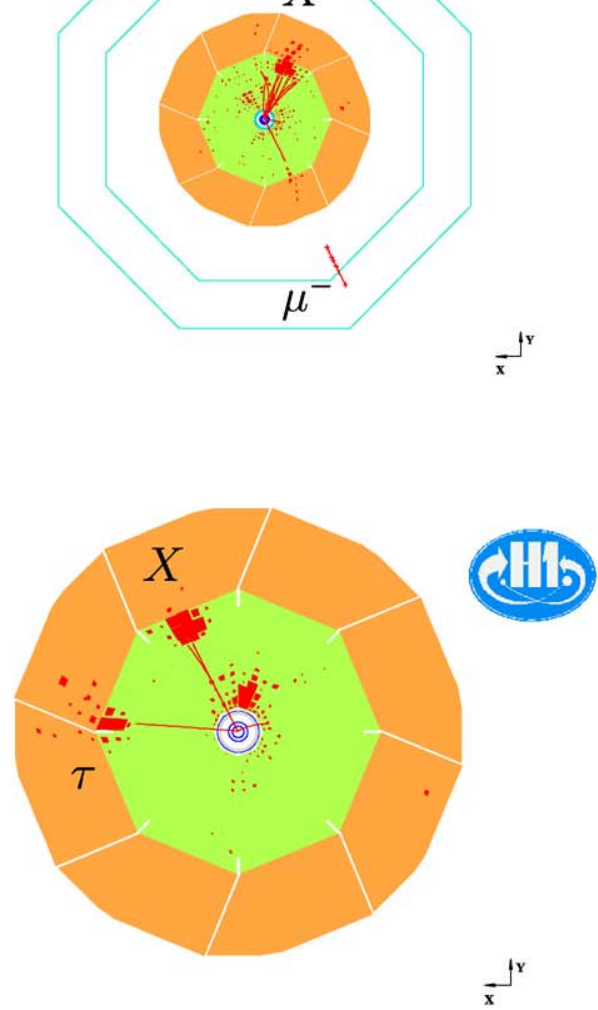

\subsection{Cross section measurements}

A measurement of the visible cross section for the isolated lepton and missing transverse momentum topology in $e p$ collisions is performed using the electron and muon channels in the phase space $5^{\circ}<\theta_{\ell}<140^{\circ}, P_{T}^{\ell}>10 \mathrm{GeV}$, $P_{T}^{\text {miss }}>12 \mathrm{GeV}$ and $D(\ell ;$ jet $)>1.0$ at a centre of mass energy $^{3}$ of $\sqrt{s}=317 \mathrm{GeV}$. The cross section is defined as:

$\sigma=\frac{N_{\mathrm{obs}}-N_{\mathrm{bg}}^{\mathrm{MC}}}{\mathcal{L} \mathcal{A}}$

\footnotetext{
${ }^{3}$ Assuming a linear dependence of the cross section on the proton beam energy.
} 
Table 4 Kinematics of the isolated lepton events at high $P_{T}^{X}>$ $25 \mathrm{GeV}$. Resolutions on the experimental observables are propagated to the reconstructed variables. The measured charge $q$ of the lepton and the associated charge significance $\sigma_{q}$ is given where appropriate. For muon events where the curvature $|\kappa| \propto 1 / P_{T}$ measurement is compatible with zero within $2 \sigma=|\kappa| / \delta \kappa$, the kinematic variables are estimated at $|\kappa|+\delta \kappa$ corresponding to a lower limit on $P_{T}$ at the $1 \sigma$ level. All events in the table are observed in $e^{+} p$ collisions with exception of the event in the last row, which was observed in $e^{-} p$ collisions

\begin{tabular}{|c|c|c|c|c|c|c|c|}
\hline \multicolumn{8}{|c|}{$\mathrm{H} 1$ isolated lepton events at high $P_{T}^{X}$} \\
\hline Run & Event & Lepton $q\left(\sigma_{q}\right)$ & $P_{T}^{\ell}[\mathrm{GeV}]$ & $\theta_{\ell}\left[{ }^{\circ}\right]$ & $P_{T}^{X}[\mathrm{GeV}]$ & $M_{T}^{\ell v}[\mathrm{GeV}]$ & $P_{T}^{\text {miss }}[\mathrm{GeV}]$ \\
\hline 186729 & 702 & $\mu$ & $>42.5$ & $30.0 \pm 0.4$ & $75.3 \pm 5.5$ & $>33.7$ & $>40.0$ \\
\hline 188108 & 5066 & $\mu^{-(8.3 \sigma)}$ & $40.9_{-4.4}^{+5.6}$ & $35.1 \pm 0.4$ & $29.4 \pm 2.4$ & $79.2_{-10.1}^{+8.0}$ & $43.7_{-4.2}^{+3.3}$ \\
\hline 192227 & 6208 & $\mu^{-(7.0 \sigma)}$ & $73.3_{-9.2}^{+12.2}$ & $28.6 \pm 0.3$ & $63.9 \pm 5.9$ & $67.8_{-24.9}^{+19.8}$ & $19.8_{-6.8}^{+5.4}$ \\
\hline 195308 & 16793 & $\mu^{+(4.2 \sigma)}$ & $60.1_{-11.5}^{+18.6}$ & $30.9 \pm 0.4$ & $30.1 \pm 2.6$ & $88.7_{-37.0}^{+23.5}$ & $33.5_{-15.8}^{+10.6}$ \\
\hline 248207 & 32134 & $e^{+(15 \sigma)}$ & $32.1 \pm 1.3$ & $32.2 \pm 0.3$ & $42.0 \pm 3.9$ & $62.7 \pm 2.3$ & $43.4 \pm 2.8$ \\
\hline 252020 & 30485 & $e^{+(40 \sigma)}$ & $25.6 \pm 1.2$ & $110.2 \pm 0.3$ & $39.1 \pm 3.3$ & $48.6 \pm 2.1$ & $35.5 \pm 2.5$ \\
\hline 266336 & 4126 & $\mu^{+(26 \sigma)}$ & $19.7_{-0.7}^{+0.8}$ & $67.3 \pm 0.4$ & $50.0 \pm 3.8$ & $69.8_{-2.5}^{+2.4}$ & $66.6 \pm 3.7$ \\
\hline 268338 & 70014 & $e^{+(1.6 \sigma)}$ & $33.8 \pm 1.3$ & $29.7 \pm 0.2$ & $45.2 \pm 3.2$ & $90.3 \pm 3.1$ & $67.2 \pm 3.0$ \\
\hline 275991 & 29613 & $e^{+(37 \sigma)}$ & $37.8 \pm 1.5$ & $41.7 \pm 0.3$ & $27.1 \pm 1.8$ & $73.3 \pm 2.8$ & $40.3 \pm 1.4$ \\
\hline 369241 & 6588 & $e$ & $29.2 \pm 1.1$ & $20.3 \pm 0.2$ & $40.5 \pm 4.8$ & $74.3 \pm 3.0$ & $55.5 \pm 4.2$ \\
\hline 385422 & 76666 & $e^{+(22 \sigma)}$ & $28.1 \pm 1.3$ & $96.1 \pm 0.3$ & $25.9 \pm 2.8$ & $63.1 \pm 2.8$ & $40.0 \pm 2.3$ \\
\hline 389826 & 2783 & $e^{-(10 \sigma)}$ & $62.0 \pm 2.2$ & $45.6 \pm 0.3$ & $45.3 \pm 4.5$ & $79.7 \pm 6.0$ & $30.3 \pm 2.1$ \\
\hline 391884 & 49715 & $e$ & $38.2 \pm 1.4$ & $22.7 \pm 0.2$ & $32.4 \pm 2.6$ & $48.5 \pm 3.0$ & $20.1 \pm 0.8$ \\
\hline 473929 & 107593 & $\mu^{-(9.6 \sigma)}$ & $53.5_{-5.1}^{+6.2}$ & $31.4 \pm 0.4$ & $49.1 \pm 4.5$ & $80.6_{-10.7}^{+8.7}$ & $40.9_{-3.4}^{+2.8}$ \\
\hline 494115 & 121996 & $\mu^{+(22 \sigma)}$ & $22.6_{-1.0}^{+1.0}$ & $61.5 \pm 0.4$ & $37.0 \pm 3.7$ & $45.2_{-1.9}^{+1.8}$ & $35.8_{-3.0}^{+3.0}$ \\
\hline 495399 & 85500 & $\mu^{-(32 \sigma)}$ & $29.4_{-0.8}^{+0.9}$ & $62.4 \pm 0.4$ & $29.6 \pm 2.8$ & $63.1_{-1.8}^{+1.7}$ & $40.3_{-2.0}^{+2.0}$ \\
\hline 498117 & 316609 & $e^{+(9.8 \sigma)}$ & $27.4 \pm 1.1$ & $30.7 \pm 0.3$ & $26.7 \pm 1.8$ & $72.5 \pm 2.5$ & $49.9 \pm 2.0$ \\
\hline 433051 & 64528 & $e^{-(24 \sigma)}$ & $26.2 \pm 1.3$ & $69.9 \pm 0.3$ & $72.9 \pm 5.6$ & $71.3 \pm 2.9$ & $75.8 \pm 5.2$ \\
\hline
\end{tabular}

Fig. 4 The hadronic transverse momentum $P_{T}^{X}$ distributions in the combined electron and muon channels for the $e^{+} p(\mathbf{a})$ and $e^{-} p$ (b) data samples. The data (points) are compared to the SM expectation (open histogram). The signal component of the SM expectation, dominated by real $W$ production, is shown as the hatched histogram. The total uncertainty on the SM expectation is shown as the shaded band

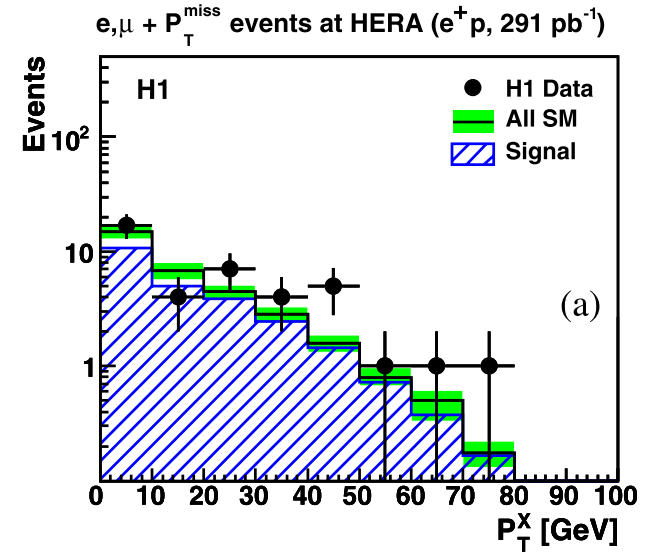

where $N_{\text {obs }}$ is the number of observed data events in the complete $e^{ \pm} p$ data set and $N_{\mathrm{bg}}^{\mathrm{MC}}$ is the MC estimate of the number of SM background events, due to processes discussed in Sect. 2. The total data luminosity is denoted by $\mathcal{L}$. The acceptance $\mathcal{A}$ is defined as $N_{\text {rec }}^{\mathrm{MC}} / N_{\text {gen }}^{\mathrm{MC}}$, the ratio of reconstructed and generated signal events, and accounts for detection efficiencies and migrations. The EPVEC generator is used to calculate the acceptance, which is predicted to be about the same for $e^{+} p$ and $e^{-} p$ collisions.
The cross section is measured in several regions of $P_{T}^{X}$ in both lepton channels, as displayed in Table 6. For $P_{T}^{X}<$ $12 \mathrm{GeV}$, the cross section in the muon channel is estimated using the electron channel measurement [52]. The measured total visible cross section for events with an isolated lepton and missing transverse momentum is:

$\sigma_{\ell+P_{T}^{\text {miss }}}=0.23 \pm 0.05$ (stat.) \pm 0.04 (sys.) pb, 
Table 5 Summary of the H1 results of the search for events with tau leptons and missing transverse momentum for the $e^{+} p$ data (291 $\left.\mathrm{pb}^{-1}\right), e^{-} p$ data $\left(183 \mathrm{pb}^{-1}\right)$ and the full HERA data set $\left(474 \mathrm{pb}^{-1}\right)$. The results are shown for the full selected sample and for the subsam- ple at $P_{T}^{X}>25 \mathrm{GeV}$. The number of observed events is compared to the SM prediction. The SM signal $\left(W \rightarrow \tau \nu_{\tau}\right)$ and the background contributions are also shown. The quoted errors contain statistical and systematic uncertainties added in quadrature

\begin{tabular}{llcccc}
\hline $\mathrm{H} 1$ & Tau channel & Data & SM expectation & SM signal & Other SM processes \\
\hline $1994-2007 e^{+} p 291 \mathrm{pb}^{-1}$ & Total & 9 & $12.3 \pm 2.0$ & $1.66 \pm 0.25$ & $10.6 \pm 1.8$ \\
& $P_{T}^{X}>25 \mathrm{GeV}$ & 0 & $0.82 \pm 0.12$ & $0.38 \pm 0.06$ & $0.44 \pm 0.06$ \\
$1999-2006 e^{-} p 183 \mathrm{pb}^{-1}$ & Total & 9 & $11.0 \pm 1.9$ & $1.00 \pm 0.15$ & $10.0 \pm 1.8$ \\
& $P_{T}^{X}>25 \mathrm{GeV}$ & 1 & $0.68 \pm 0.11$ & $0.21 \pm 0.03$ & $0.47 \pm 0.07$ \\
$1994-2007 e^{ \pm} p 474 \mathrm{pb}^{-1}$ & Total & 18 & $23.2 \pm 3.8$ & $2.66 \pm 0.40$ & $20.6 \pm 3.4$ \\
& $P_{T}^{X}>25 \mathrm{GeV}$ & 1 & $1.50 \pm 0.21$ & $0.59 \pm 0.09$ & $0.91 \pm 0.12$ \\
\hline
\end{tabular}

Fig. 5 Distributions in the tau channel for the $e^{ \pm} p$ data sample. Shown is the missing transverse momentum $P_{T}^{\text {miss }}(\mathbf{a})$, the hadronic transverse momentum not including the tau-jet candidate $P_{T}^{X^{\prime}}(\mathbf{b})$, the polar angle of the tau-jet candidate $\theta_{\tau}(\mathbf{c})$ and the tau-jet candidate transverse momentum $P_{T}^{\tau}(\mathbf{d})$. The data (points) are compared to the SM expectation (open histogram). The signal component of the SM expectation is shown as the hatched histogram. The total uncertainty on the SM expectation is shown as the shaded band

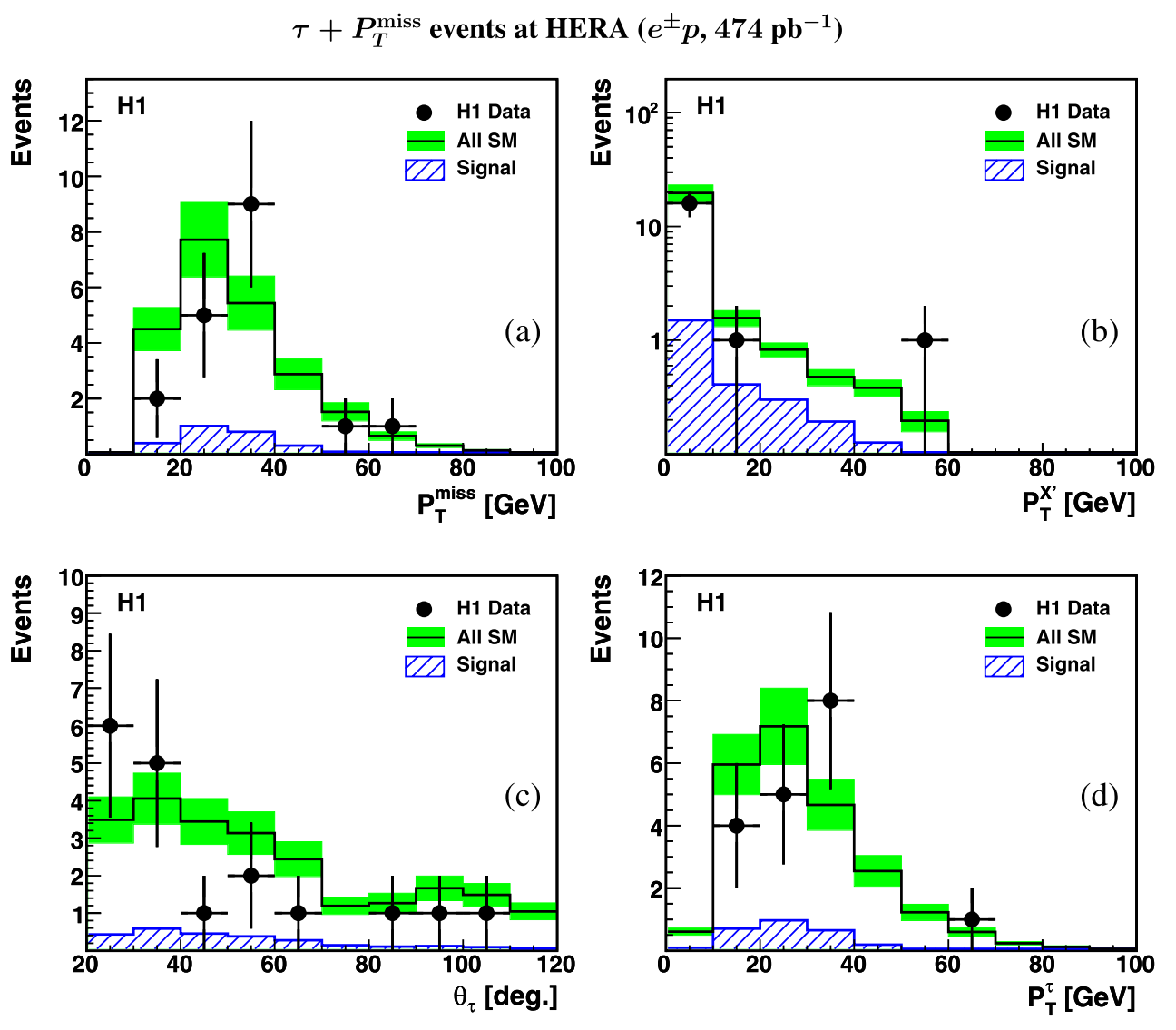

where the first error is statistical and the second systematic. The total cross section is in agreement with the SM NLO value of $0.25 \pm 0.04 \mathrm{pb}$. The results are also found to be in agreement with previously published numbers [3]. The measurements in the electron and muon channels are consistent with each other.

The systematic uncertainty includes all of the sources described in Sect. 6. In addition, a $10 \%$ model uncertainty is applied to the acceptance, estimated by comparing two further generators that produce $W$ bosons with different kinematic distributions from those in EPVEC, namely an im- plementation of $W$ boson production within PYTHIA and a flavour changing neutral current (FCNC) single top generator ANOTOP [4].

The total single $W$ boson production cross section is also calculated using (1), where $\mathcal{A}$ is now defined with respect to the full phase space and the contribution from $Z$ production illustrated in Fig. 1(d) is considered as background. The branching ratio corresponding to the leptonic $W$ boson decay to any final state with an electron or muon, including the contribution from leptonic tau-decay [53], is now also 
Table 6 The measured cross sections with statistical (stat.) and systematic (sys.) errors for events with an isolated high energy electron or muon with missing transverse momentum, in different kinematic regions of the hadronic transverse momentum $P_{T}^{X}$. The cross sections are determined for the kinematic region: $5^{\circ}<\theta_{\ell}<140^{\circ}, P_{T}^{\ell}>$
$10 \mathrm{GeV}, P_{T}^{\text {miss }}>12 \mathrm{GeV}$ and $D(\ell$; jet $)>1.0$ at a centre of mass energy of $\sqrt{s}=317 \mathrm{GeV}$. Also shown are the signal expectations including the theoretical uncertainties for the Standard Model where the dominant contribution $e p \rightarrow e W X$ is calculated at next to leading order (SM NLO)

H1 isolated lepton and $P_{T}^{\text {miss }}$ cross section

\begin{tabular}{llcc}
\hline & & Measured \pm stat. \pm sys. [fb] & SM NLO [fb] \\
\hline Electron & $P_{T}^{X} \leq 12 \mathrm{GeV}$ & $63 \pm 22 \pm 13$ & $84 \pm 13$ \\
& $P_{T}^{X}>12 \mathrm{GeV}$ & $54 \pm 17 \pm 9$ & $49 \pm 7$ \\
Muon & $P_{T}^{X}>12 \mathrm{GeV}$ & $56 \pm 16 \pm 7$ & $44 \pm 7$ \\
Combined & $P_{T}^{X} \leq 25 \mathrm{GeV}$ & $164 \pm 45 \pm 32$ & $207 \pm 31$ \\
& $P_{T}^{X}>25 \mathrm{GeV}$ & $64 \pm 18 \pm 10$ & $47 \pm 7$ \\
& Total & $228 \pm 48 \pm 39$ & $253 \pm 38$ \\
\hline
\end{tabular}

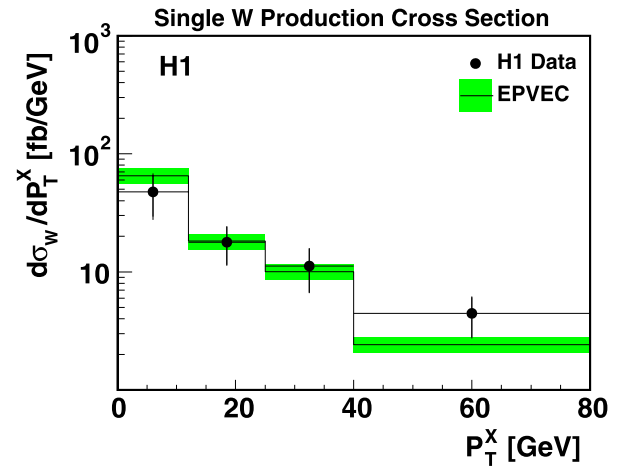

Fig. 6 The measured differential single $W$ boson production cross section $\mathrm{d} \sigma_{W} / \mathrm{d} P_{T}^{X}$ as a function of the hadronic transverse momentum $P_{T}^{X}$ (points). The errors denote the sum of the statistical and systematic uncertainties in quadrature. The measurement is compared to the EPVEC prediction (open histogram), including the theoretical uncertainty of $15 \%$ shown as the shaded band

included in the calculation. The total single $W$ boson production cross section at HERA is measured as:

$\sigma_{W}=1.14 \pm 0.25$ (stat.) \pm 0.14 (sys.) pb,

in good agreement with the SM expectation of $1.27 \pm$ $0.19 \mathrm{pb}$. This result is also in good agreement with the ZEUS measurement presented in [12]. The differential single $W$ boson production cross section is also measured as a function of $P_{T}^{X}$, the results of which are displayed in Fig. 6 and Table 7, and is in agreement with the SM prediction.

\subsection{Measurement of the $W W \gamma$ couplings}

The production of single $W$ bosons at HERA is sensitive to anomalous triple gauge boson couplings [54], via the process illustrated in Fig. 1(b). Due to the large mass of the $Z$ boson, no sensitivity is expected to the $W W Z$ coupling. Under the assumption of charge and parity conservation, and
Table 7 The differential single $W$ boson production cross section $\mathrm{d} \sigma_{W} / \mathrm{d} P_{T}^{X}$ with statistical (stat.) and systematic (sys.) errors, derived from the electron and muon channels as a function of the hadronic transverse momentum $P_{T}^{X}$. Also shown are the expectations including the theoretical uncertainties for the Standard Model calculated at next to leading order (SM NLO). In the phase space where $P_{T}^{X}<12 \mathrm{GeV}$, the electron measurement is used to estimate the muon cross section under the assumption of lepton universality

\begin{tabular}{lcc}
\hline \multicolumn{3}{l}{ H1 differential single $W$ production cross section at $\sqrt{s}=317 \mathrm{GeV}$} \\
\hline$P_{T}^{X}[\mathrm{GeV}]$ & Measured \pm stat. \pm sys. $[\mathrm{fb} / \mathrm{GeV}]$ & SM NLO $[\mathrm{fb} / \mathrm{GeV}]$ \\
\hline $0-12$ & $47.6 \pm 18.2 \pm 8.9$ & $65.2 \pm 9.8$ \\
$12-25$ & $17.4 \pm 6.3 \pm 1.7$ & $18.3 \pm 2.7$ \\
$25-40$ & $11.2 \pm 4.5 \pm 1.0$ & $10.1 \pm 1.5$ \\
$40-80$ & $4.5 \pm 1.7 \pm 0.6$ & $2.4 \pm 0.4$ \\
\hline
\end{tabular}

Lorentz and electromagnetic gauge invariance, the $W W \gamma$ vertex can be parametrised [55] using two free coupling parameters, $\kappa$ and $\lambda$, related to the magnetic dipole moment $\mu_{W}=e / 2 M_{W}(1+\kappa+\lambda)$ and the electric quadrupole moment $Q_{W}=-e / M_{W}^{2}(\kappa-\lambda)$ of the $W$ boson. In the SM, $\kappa=1$ and $\lambda=0$ at tree level. In this paper, limits are set on the coupling parameters using the method of maximum likelihood in a Bayesian approach employing Poisson statistics. Instead of $\kappa, \Delta \kappa \equiv \kappa-1$ is used, such that any non-zero value for $\Delta \kappa$ or $\lambda$ represents a deviation from the SM.

Since the $P_{T}^{X}$ spectrum is expected to be sensitive to anomalous values of $\Delta \kappa$ and $\lambda$ [54], the likelihood analysis is performed in four bins with lower edges in $P_{T}^{X}$ of $12,25,40$ and $80 \mathrm{GeV}$. The last bin extends until $120 \mathrm{GeV}$ and contains no data events, but for anomalous values of $\lambda$, the expectation in this region becomes significant and is used to further constrain $\lambda$. Events with $P_{T}^{X}<12 \mathrm{GeV}$ are not used since the main SM $W$ boson production diagram, shown in Fig. 1(a), dominates this region of phase space and no sensitivity to the $W W \gamma$ vertex parameters is gained from such events. 
Fig. 7 Probability distributions (in arbitrary units) of the single parameter fits to (a) $\Delta \kappa$ and (b) $\lambda$. The obtained $95 \% \mathrm{CL}$ limits are shown (shaded areas) in comparison with those of DØ [61], CDF [62], and ALEPH [63]. The SM expectation values are also indicated

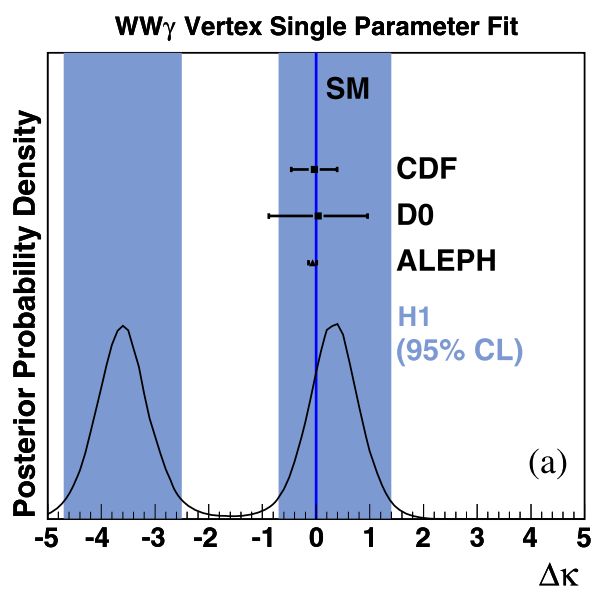

WW $\gamma$ Vertex Single Parameter Fit

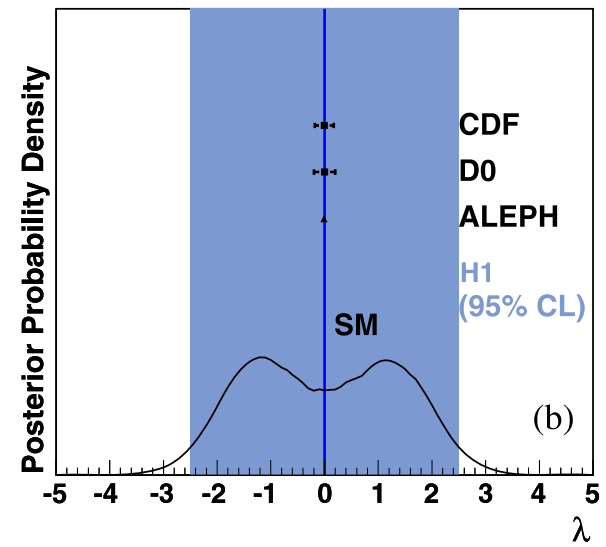

The maximum likelihood analysis is performed for each bin in $P_{T}^{X}$ and the resulting probability distributions are multiplied. This is done for $\Delta \kappa$ and $\lambda$ separately, keeping the other parameter fixed to its SM value. The resulting probability distributions are shown in Fig. 7. The observed double-peak structure in the probability densities arises from the quadratic dependence of the cross section to the coupling parameters. The following limits are derived at 95\% confidence level (CL):

$-4.7<\Delta \kappa<-2.5$ or $\quad-0.7<\Delta \kappa<1.4$,

$-2.5<\lambda<2.5$.

The dominant source of error is the theoretical uncertainty on the single $W$ boson production cross section. The limits obtained are in good agreement with the SM prediction. Since the value of $\Delta \kappa=-1$ is excluded at $95 \% \mathrm{CL}$, the results obtained explicitly demonstrate the presence of a magnetic coupling of the photon to the $W$ boson, in addition to the coupling to the electric charge of the $W$ boson. Given the limits obtained for $\lambda$, the first allowed region $(-4.7<\Delta \kappa<-2.5)$ is excluded by limits derived from loop contributions to $(g-2)_{\mu}$ [56-60]. The limits on $\Delta \kappa$, enclosing the SM prediction $\Delta \kappa=0$, are compatible with those obtained at the Tevatron $[61,62]$. The most stringent limits were obtained at LEP in single $\gamma$, single $W$ and $W$ pair production [63-66].

\subsection{Measurement of the $W$ boson polarisation fractions}

A measurement of the $W$ boson polarisation is performed to further test the compatibility of the observed results with SM single $W$ production. For $W$ bosons from the decay of single top quarks, the production of which is kinematically possible at HERA, the polarisation is expected to be different from that in the SM. The $\cos \theta^{*}$ distribution in the decay $W \rightarrow e / \mu+v$ is exploited, where $\theta^{*}$ is defined as the angle between the $W$ boson momentum in the lab frame and that of the charged decay lepton in the $W$ boson rest frame. The influence of higher order corrections on the $\cos \theta^{*}$ distribution is expected to be small and is neglected. For the left-handed polarisation fraction $F_{-}$, the longitudinal fraction $F_{0}$ and the right-handed fraction $F_{+} \equiv 1-F_{-}-F_{0}$, the $\cos \theta^{*}$ distributions for $W^{+}$bosons are given [55] by:

$$
\begin{aligned}
& \frac{1}{\sigma_{W \rightarrow \ell+v}} \frac{\mathrm{d} \sigma_{W \rightarrow \ell+v}}{\mathrm{~d} \cos \theta^{*}} \\
& =\frac{3}{4} F_{0}\left(1-\cos ^{2} \theta^{*}\right)+\frac{3}{8} F_{-}\left(1-\cos \theta^{*}\right)^{2} \\
& \quad+\frac{3}{8} F_{+}\left(1+\cos \theta^{*}\right)^{2} .
\end{aligned}
$$

To allow the datasets of both $W$ boson charges to be combined, $\cos \theta^{*}$ is multiplied by the sign of the lepton charge $q_{\ell}$. Events in the electron or muon channel originating from $W$ boson to tau decay, $W \rightarrow \tau\left(\rightarrow e / \mu+v_{e / \mu}\right)+$ $\nu_{\tau}$, are considered background, since for these events the $\cos \theta^{*}$ distributions are not expected to be described by (2).

Events are selected for this measurement starting from the sample described in Sect. 5.1. The $W$ boson four-vector is reconstructed as the sum of the neutrino and lepton fourvectors using a kinematic constraint to the $W$ mass if the scattered electron is not observed [52]. To ensure a reliable charge measurement only leptons in the central region $\theta_{\ell}>20^{\circ}$, and with charge significance $\sigma_{q}>1$ are considered. In the electron channel, the associated track transverse momentum $P_{T}^{\text {track }}$ is required to match the calorimetric measurement $P_{T}^{e}$ under the condition $1 / P_{T}^{\text {track }}-1 / P_{T}^{e}<0.04$. Using these requirements the charge misidentification probability is found to be below $1 \%$. The final sample consists of 21 electron events and 9 muon events. The SM prediction is dominated by single $W$ production, which contributes $76 \%$ to the total expectation.

The $\cos \theta^{*}$ distributions of $W$ bosons produced off-shell are not expected to be described by the polarisation model given in (2). A correction is therefore applied to the measured $q_{\ell} \cdot \cos \theta^{*}$ distribution [52], where the contribution 


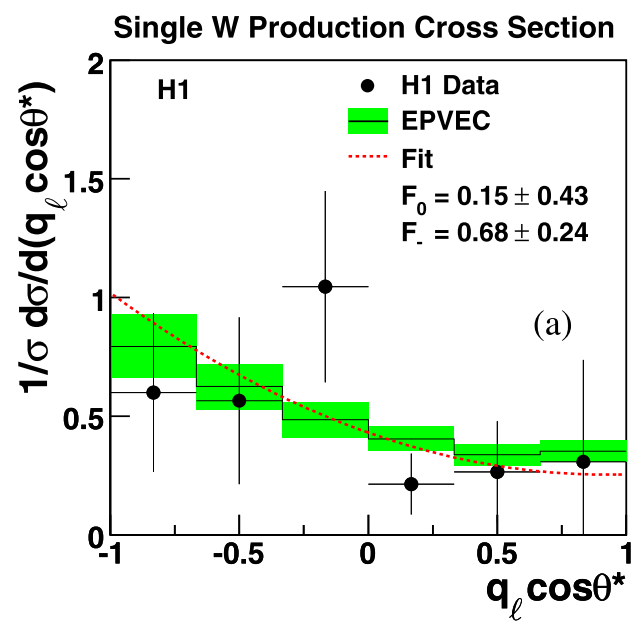

Fig. 8 (a) The measured normalised differential cross section $1 / \sigma d \sigma / d\left(q_{\ell} \cdot \cos \theta^{*}\right)$ (points) as a function of $q_{\ell} \cdot \cos \theta^{*}$ for on-shell $W$ bosons. The EPVEC prediction is also shown (open histogram) with a $15 \%$ theoretical uncertainty shown by the band. The result of the simultaneous fit of the $W$ polarisation fractions is shown as the dashed

Table 8 The polarisation fractions $F_{-}$and $F_{0}$ obtained from one parameter fits, with statistical (stat.) and systematic (sys.) errors. The central values are obtained by fixing one parameter to its SM prediction and fitting the other. The SM predictions from EPVEC are also shown

H1 $W$ polarisation fractions

\begin{tabular}{lll}
\hline Fraction & Measured \pm stat. \pm sys. & EPVEC \\
\hline$F_{-}$ & $0.67 \pm 0.19 \pm 0.08$ & 0.62 \\
$F_{0}$ & $0.21 \pm 0.35 \pm 0.10$ & 0.17 \\
\hline
\end{tabular}

from such production diagrams is estimated using EPVEC. The measured $q_{\ell} \cdot \cos \theta^{*}$ distribution is also corrected for acceptance and detector effects. The resulting normalised differential cross section is shown in Fig. 8(a), with the result of the fit to the polarisation fractions described in the above equation. In the fit, $F_{-}$and $F_{0}$ are simultaneously determined, the result of which is shown in Fig. 8(b) and found to be in good agreement with the SM. To test the stability or possible biases of the result, the fit is repeated on $\cos \theta^{*}$ distributions derived using non-SM polarisation fractions. As a further cross check, a fit to the uncorrected $\cos \theta^{*}$ distribution using polarisation templates is performed on detector level. All results are found to be consistent.

In addition, Fig. 8(b) shows the expectation obtained from ANOTOP [4], where all $W$ bosons are assumed to come from top decays. The data are also compatible with this process within the sensitivity of the measurement, although it can be seen that the SM value is favoured.

$F_{-}$and $F_{0}$ are also extracted in fits where one parameter is fixed to its SM value, the results of which are presented in Table 8 . The quoted systematic uncertainties are propagated from the cross section calculations. The systematic effects

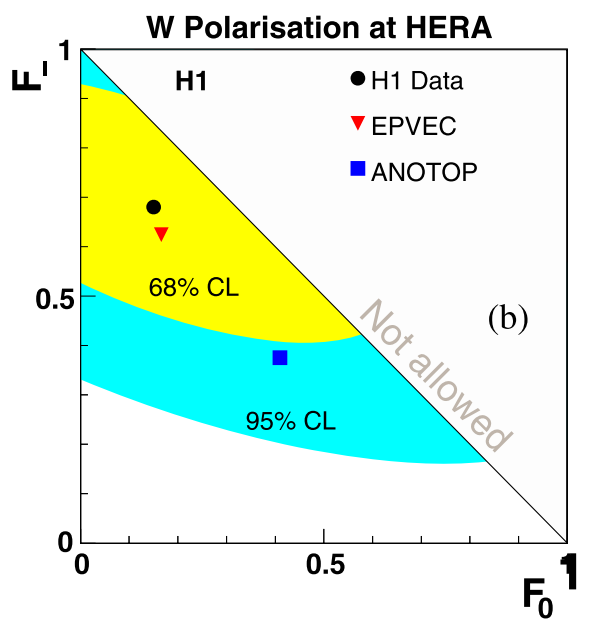

histogram. (b) The plane showing the fit result for the simultaneously extracted left handed $\left(F_{-}\right)$and longitudinal $\left(F_{0}\right) W$ boson polarisation fractions (point) with the corresponding $68 \%$ and $95 \%$ CL contours. Also shown are the values of predictions from EPVEC (triangle) and ANOTOP (square)

on the shape of the differential cross section from uncertainties on the background estimates and charge misidentification are also taken into account. More details on the analysis can be found in [52].

\section{Summary}

A search for events with isolated leptons and missing transverse momentum is performed using the full HERA $e^{ \pm} p$ data sample, corresponding to an integrated luminosity of $474 \mathrm{pb}^{-1}$. All three lepton flavours are investigated. With respect to the previous publication the integrated luminosity of the data set is increased by about a factor of four, and the signal efficiency and background suppression have been improved.

In the combined electron and muon channels 53 events are observed in the full $e^{ \pm} p$ data sample compared to a SM prediction of $54.1 \pm 7.4$, where $40.4 \pm 6.3$ are expected from signal processes, dominated by single $W$ production. Interesting events are observed at high $P_{T}^{X}>25 \mathrm{GeV}$ in the $e^{+} p$ data sample, where 17 events are observed compared to a SM prediction of $8.0 \pm 1.3$, including $7.0 \pm 1.2$ expected from signal processes. This excess of data events over the SM prediction in the $e^{+} p$ data corresponds to a $2.4 \sigma$ significance, which is lower than previously reported in [3]. No such excess is observed in the $e^{-} p$ data.

In the search for tau leptons and missing transverse momentum, 18 events are observed in agreement with the SM expectation of $23.2 \pm 3.8$, which is dominated by CC background. The signal contribution is small at around $11 \%$. No excess of events is observed in the data at large hadronic transverse momentum. 
From the events observed in the electron and muon channels the single $W$ production cross section has been measured as $1.14 \pm 0.25$ (stat.) \pm 0.14 (sys.) pb, compared to a $\mathrm{SM}$ expectation of $1.27 \pm 0.19 \mathrm{pb}$. The measured cross section is used to derive single parameter limits on the $W W \gamma$ gauge coupling parameters $\Delta \kappa$ and $\lambda$ at $95 \%$ CL. The $W$ polarisation fractions are measured for the first time at HERA and found to be consistent with the SM.

Acknowledgements We are grateful to the HERA machine group whose outstanding efforts have made this experiment possible. We thank the engineers and technicians for their work in constructing and maintaining the $\mathrm{H} 1$ detector, our funding agencies for financial support, the DESY technical staff for continual assistance and the DESY directorate for support and for the hospitality which they extend to the non-DESY members of the collaboration.

\section{Appendix A: Background studies}

The description of the SM background and the potential influence of systematic effects arising from the event selection are investigated using a set of control samples, where individual background processes are statistically enriched. In particular, the origin of features of the final sample, such as the difference in event yield in the $e^{+} p$ and $e^{-} p$ data have been examined. The control samples are defined by removing cuts from the event selections summarised in Tables 1 and 2. The most conclusive background enriched samples are described in the following.

\section{A.1 Electron channel control samples}

The SM background in the electron channel is studied by enriching the final sample with $\mathrm{NC}$ and $\mathrm{CC}$ events.

NC background, concentrated at low $Q_{e}^{2}$, may enter the final sample due to a mismeasurement of energy which gives rise to a large value of $P_{T}^{\text {calo }}$ and a small value of $V_{\mathrm{ap}} / V_{\mathrm{p}}$. The requirements on $\zeta_{e}^{2}, P_{T}^{\text {calo }}$ and $V_{\text {ap }} / V_{\mathrm{p}}$ are designed to suppress NC that enters due to this effect. Figure 9 shows a NC enriched electron sample that is formed by removing these requirements from the final selection. The NC contribution to the SM expectation is about $95 \%$ in this sample. Figures 9(a) and (b) show the $P_{T}^{\text {calo }}$ distribution, and (c) and (d) the $\zeta_{e}^{2}$ distribution under the condition $P_{T}^{\text {calo }}<25 \mathrm{GeV}$, in the $e^{+} p$ data and in the $e^{-} p$ data, respectively. It can be seen that the part of the sample populated by the NC background at low $P_{T}^{\text {calo }}$ and low $\zeta_{e}^{2}$ is well described by the simulation for both the $e^{+} p$ data and the $e^{-} p$ data. Events in the final sample appear at $P_{T}^{\text {calo }}>25 \mathrm{GeV}$ or $\zeta_{e}^{2}>5000 \mathrm{GeV}$.

$\mathrm{CC}$ background may enter the final sample due to hadrons or photons misidentified as isolated electrons. Such electron candidates appear predominantly close to the jet and generally do not fulfill the strict isolation and tracking
Fig. 9 Distributions of data (points) selected in the NC enriched sample in the electron channel. Shown are $P_{T}^{\text {calo }}$ and $\zeta_{e}^{2}$, the latter for events with $P_{T}^{\text {calo }}<25 \mathrm{GeV}$ in the $e^{+} p(\mathbf{a}, \mathbf{c})$ and $e^{-} p(\mathbf{b}, \mathbf{d})$ data. The total uncertainty on the SM expectation (open histogram) is shown as the shaded band. The signal component is shown as the hatched histogram. The final sample populates the region $P_{T}^{\text {calo }}>25 \mathrm{GeV}$ or $\zeta_{e}^{2}>5000 \mathrm{GeV}$
Electron Channel, NC Enriched
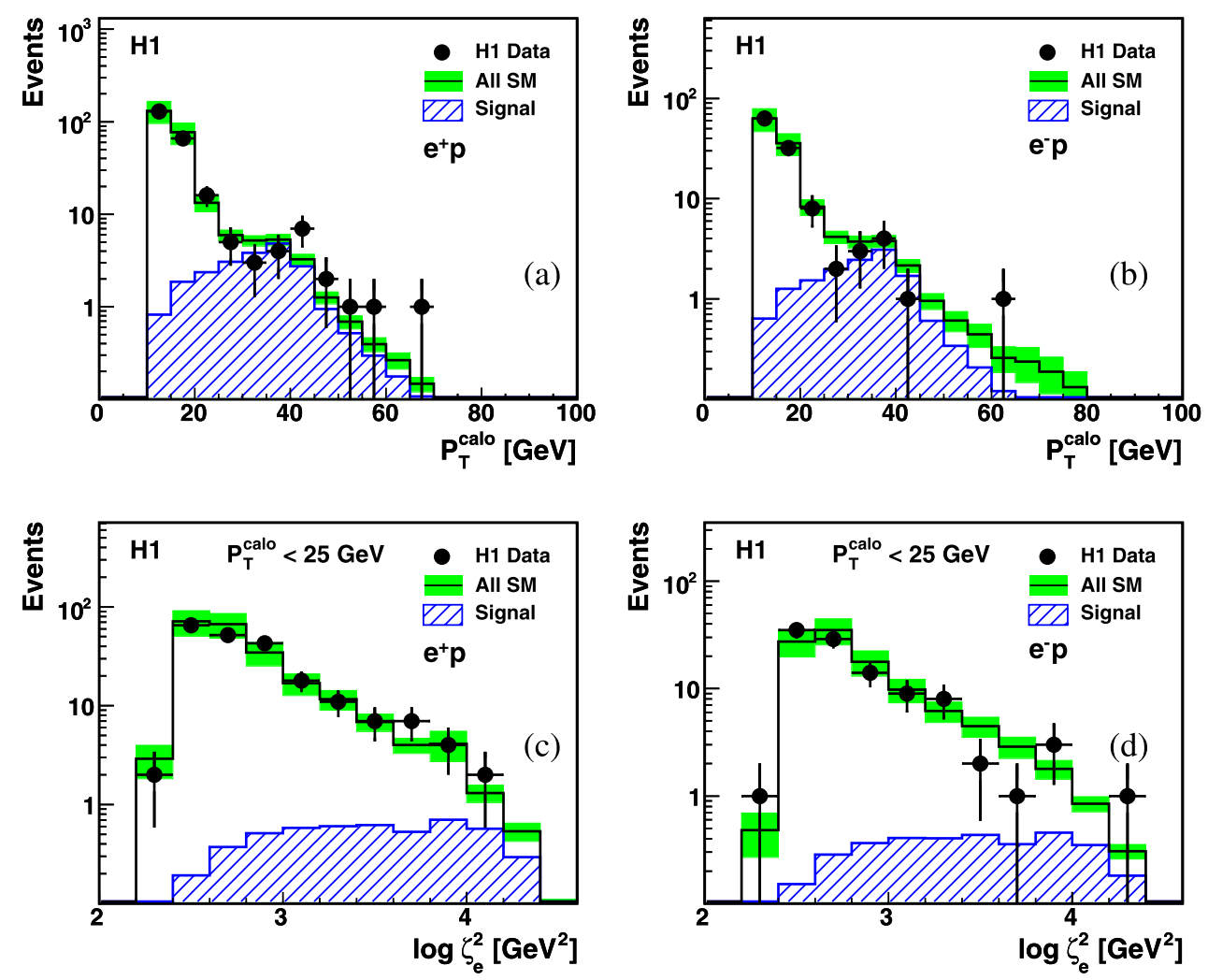
Fig. 10 Distributions of data (points) selected in the $\mathrm{CC}$ enriched sample in the electron channel. Shown is the transverse momentum $P_{T}^{e}(\mathbf{a})$ and polar angle $\theta_{e}(\mathbf{b})$ of reconstructed electrons for all data $e^{ \pm} p$, and their distance to jets

$D(e ;$ jet $)>1.0$ in the $e^{+} p(\mathbf{c})$ and $e^{-} p(\mathbf{d})$ samples individually. The total uncertainty on the SM expectation (open histogram) is shown as the shaded band. The signal component is shown as the hatched histogram. The final sample populates the region $D(e ;$ jet $)>1.0$
Electron Channel, CC Enriched
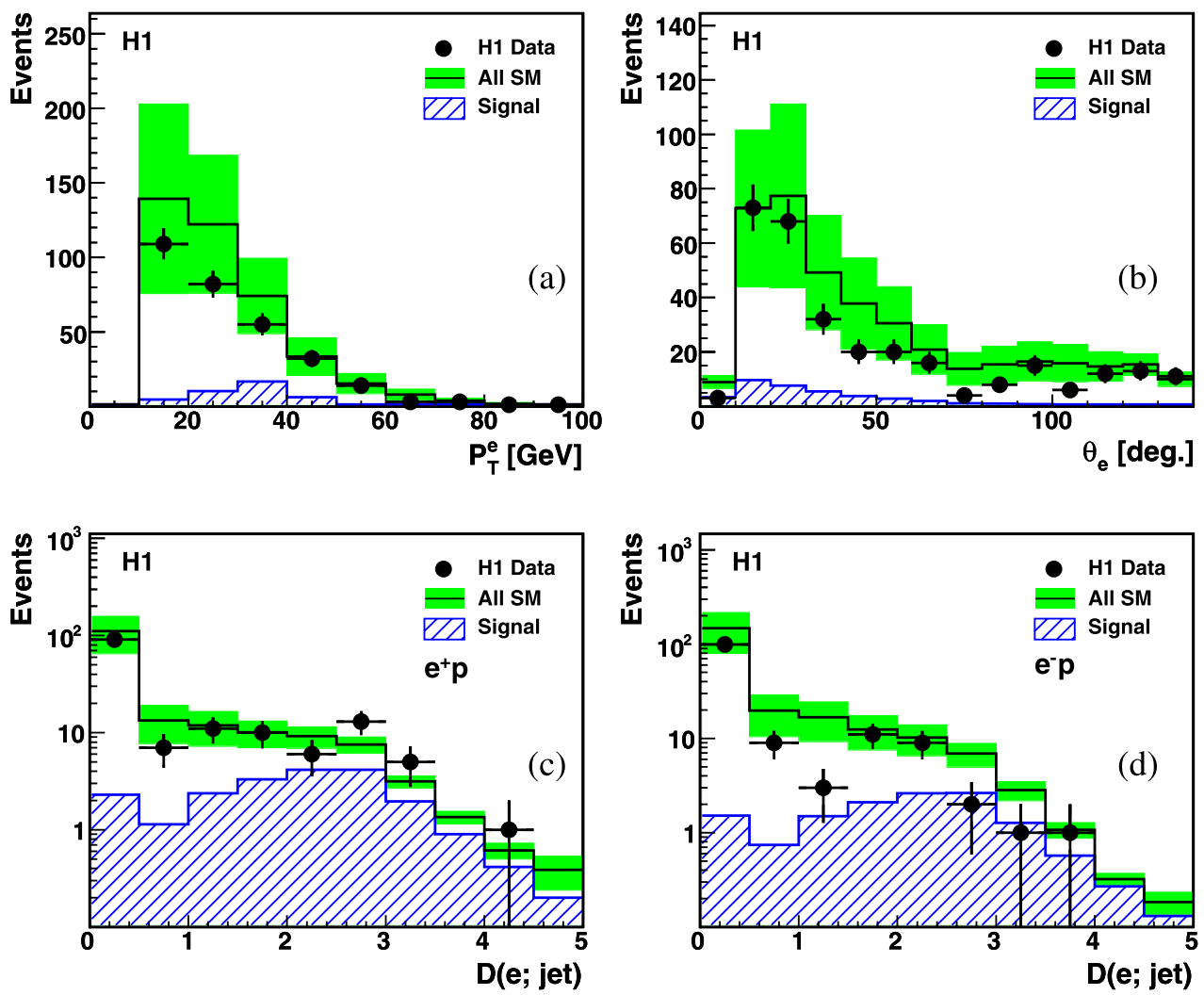

criteria applied to electrons in the final sample of this analysis. Figure 10 shows a $\mathrm{CC}$ enriched electron sample where the cuts on $D(e$; jet $)$ and $D(e$; track) are removed and only an associated NV track with a DCA $<12 \mathrm{~cm}$ is required as a veto against neutral particles. The electron candidates are still required to be isolated in the calorimeter, as described in Sect. 4. The tracking requirements on the electron are relaxed so that the overall $\mathrm{CC}$ contribution to the SM expectation is about $80 \%$ in this selection. $P_{T}^{e}$ and $\theta_{e}$ distributions of electron candidates in this sample are shown in Figs. 10(a) and (b), respectively. The model uncertainty on this type of CC background is $50 \%$, as derived from this control sample, such that the rate of isolated electrons over the whole range is described within the total uncertainties. Figures $10(\mathrm{c})$ and (d) show the $D(e$; jet) distribution in the $e^{+} p$ and $e^{-} p$ data, respectively. The CC background peaks close to the jet and is described within the total error in both data sets.

\section{A.2 Muon channel control samples}

Studies of the SM background in the muon channel are performed by enriching the final sample with lepton pair and CC events.

Muons in pair production events typically have asymmetric transverse momenta. If one of the muons is produced at a very low polar angle, it may escape detection due to geometrical acceptance. In this case the detected muon tends to balance the hadrons in the transverse plane. Figure 11 shows a muon pair enriched sample of events that is formed by removing the requirements on $P_{T}^{\text {miss }}$ and $\Delta \phi_{\mu-X}$ from the final muon selection. The requirement that there be only one muon in the event is also removed. The overall lepton pair contribution to the SM expectation is about $90 \%$ in this sample. The $P_{T}^{\text {miss }}$ distribution in this sample is shown in Fig. 11(a), and demonstrates that the resolution tail is described within the errors. The $\theta_{\mu}$ distribution shown in Fig. 11(b) follows the prediction from the SM expectation. The $\Delta \phi_{\mu-X}$ distribution is shown separately for the $e^{+} p$ data and $e^{-} p$ data in Figs. 11(c) and (d), respectively, and is described within the errors in the region populated by lepton pair production. Events in the final sample are visible in the region $\Delta \phi_{\mu-X}<170^{\circ}$, where the muon pair prediction is low. Most of these events are at values of $\Delta \phi_{\mu-X}$ that deviate from $180^{\circ}$ significantly.

Isolated muons can also appear in CC events, either due to the presence of real muons produced in the fringe of the jet decay cascade, or due to misidentification of muons as a combination of tracks from charged hadrons and noise in the muon systems. Figure 12 shows a CC enriched event sample in the muon channel that is formed by removing all isolation requirements on muon candidates, in particular cuts 
Fig. 11 Distribution of data (points) selected in the lepton pair enriched sample in the muon channel. Shown is the missing transverse momentum $P_{T}^{\text {miss }}$ (a) and polar angle $\theta_{\mu}(\mathbf{b})$ of muons for all data $e^{ \pm} p$, and the acoplanarity $\Delta \phi_{\mu-X}$ in the $e^{+} p$ (c) and $e^{-} p(\mathbf{d})$ samples individually. The total uncertainty on the SM expectation (open histogram) is shown as the shaded band. The signal component is shown as the hatched histogram. The final sample populates the region $\Delta \phi_{\mu-X}<170^{\circ}$
Fig. 12 Distribution of data (points) selected in the CC enriched sample in the muon channel. Shown is the transverse momentum $P_{T}^{\mu}(\mathbf{a})$, polar angle $\theta_{\mu}$ (b) of identified muons, the distance to jets $D(\mu$; jet) (c) and the acoplanarity $\Delta \phi_{\mu-X}$ (d) for all data. The total uncertainty on the SM expectation (open histogram) is shown as the shaded band. The signal component is shown as the hatched histogram. The final sample populates the region $D(\mu ;$ jet $)>1.0$
Muon Channel, Lepton Pair Enriched
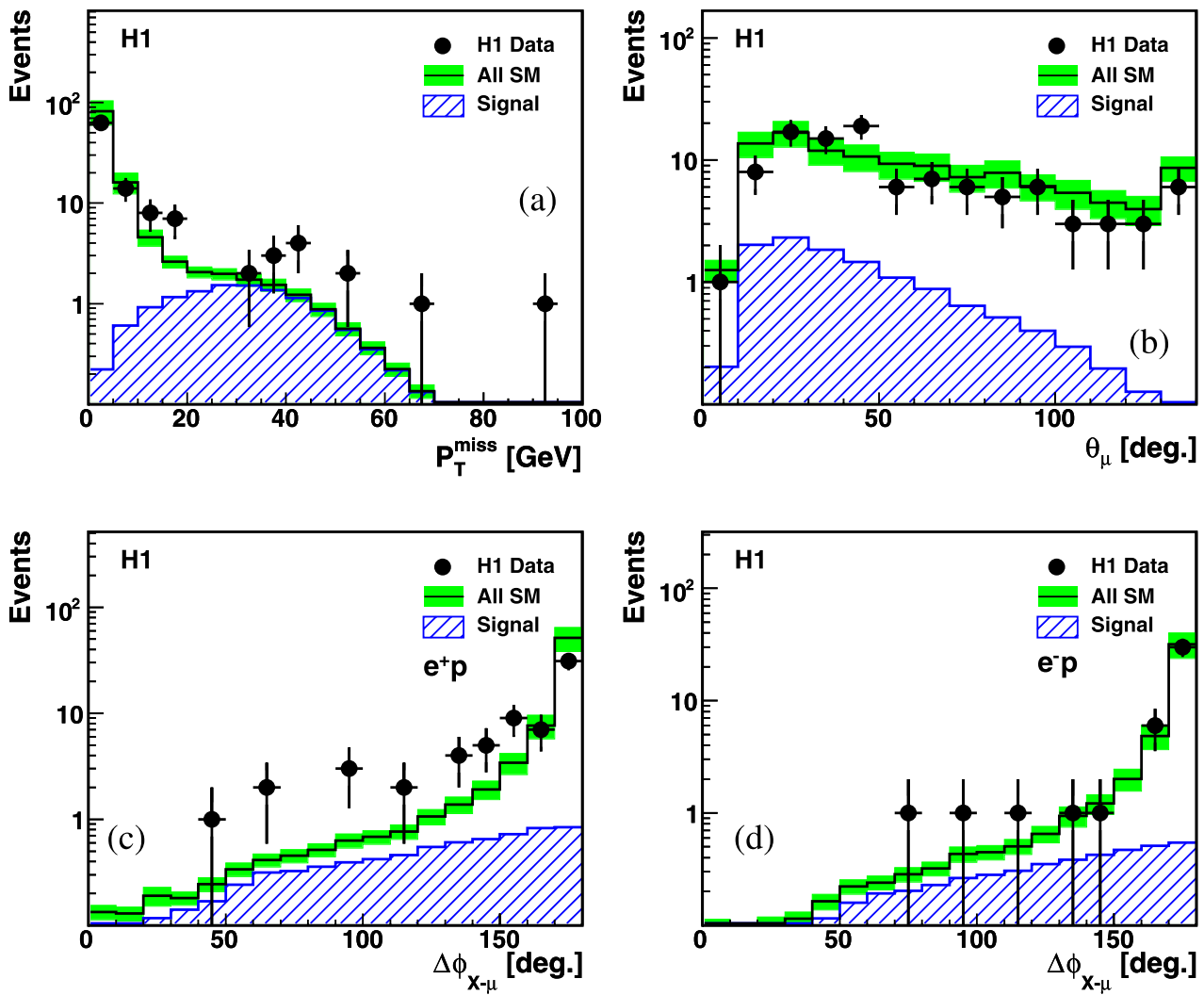

Muon Channel, CC Enriched
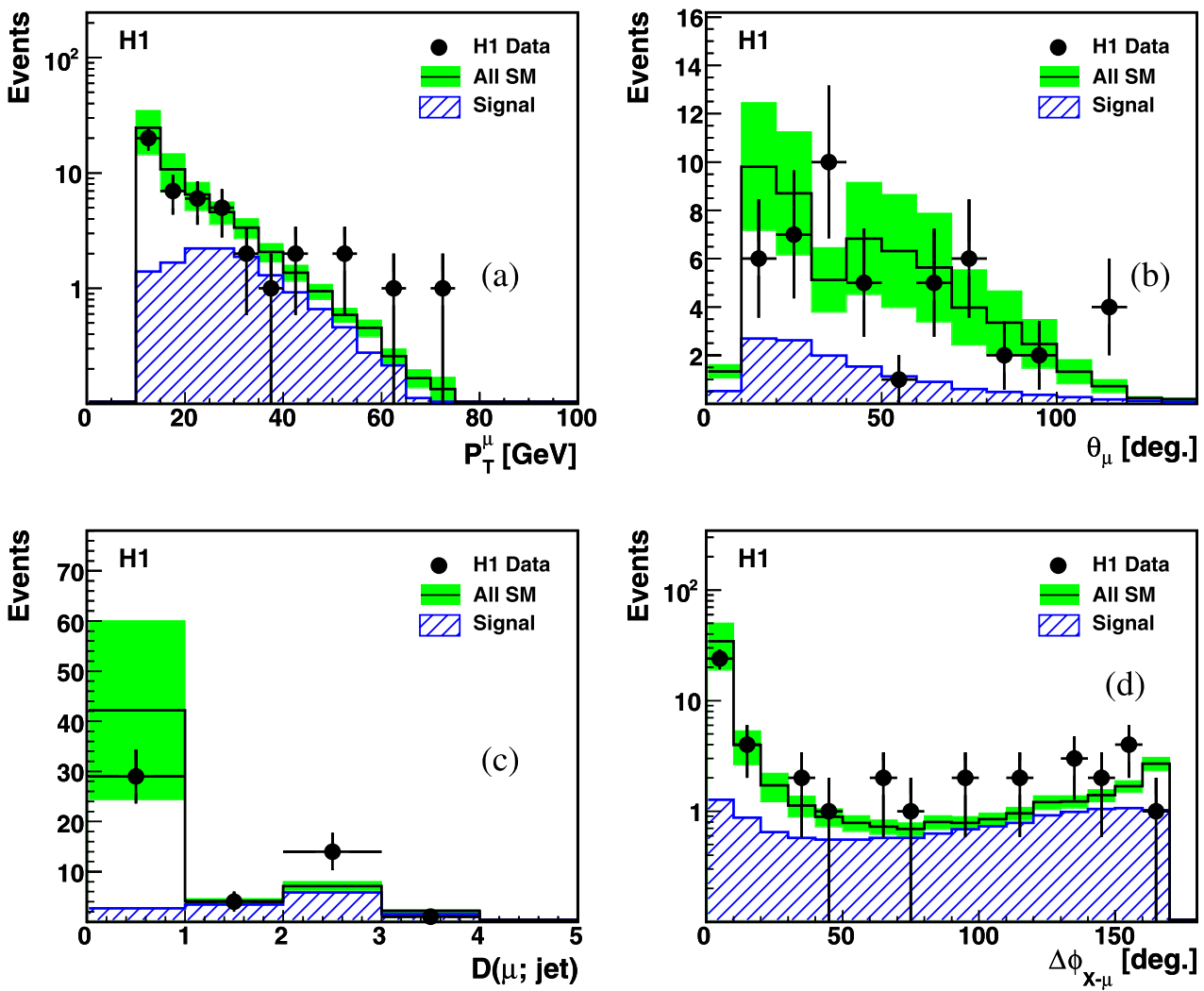
Fig. 13 Control distributions of data (points) in the tau channel. Shown is the polar angle distribution $\theta_{\text {jet }}$ of tau-like jets in an inclusive $\mathrm{NC}$ control sample (a), in a photoproduction control sample (b), in the CC-like sample (c), and the multiplicity of $P_{T}>5 \mathrm{GeV}$ tracks associated to the jet in the CC-like sample (d). The total uncertainty on the SM expectation (open histogram) is shown as the shaded band
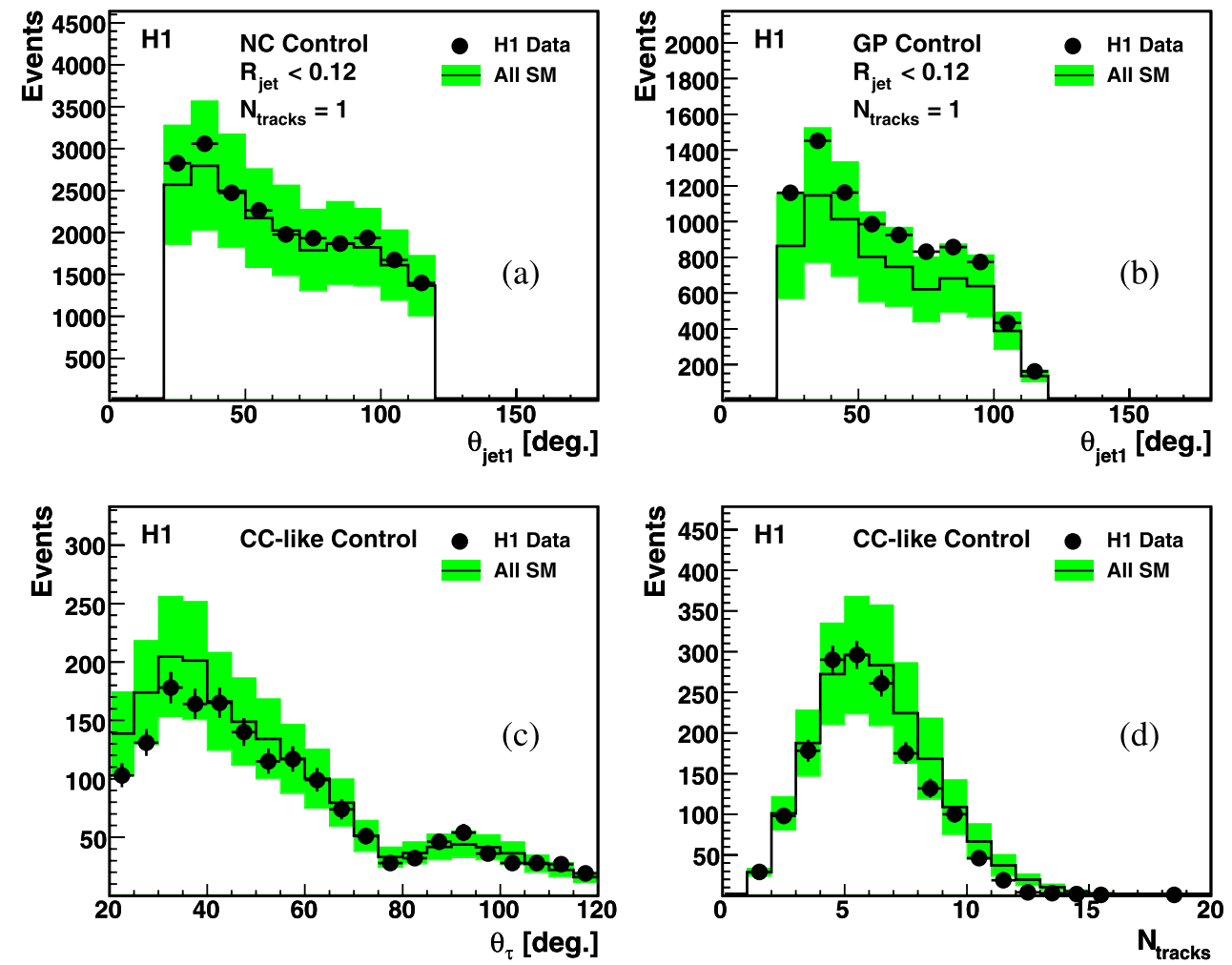

on $D(\mu$; jet $)$ and $D(\mu$; track $)$. In this sample, the contribution from $\mathrm{CC}$ to the total expectation is about $64 \%$, with the remainder dominated by signal processes. Figures 12 (a) and (b) show that $P_{T}^{\mu}$ and $\theta_{\mu}$, respectively, of identified muons in this sample follow the distribution predicted by the simulation. The rate of identified muons close to the jet is described within the total uncertainties. The $D(\mu$; jet $)$ distribution is shown in Fig. 12(c). Events in the final sample are distributed across the $\Delta \phi_{\mu-X}$ distribution, shown in Fig. 12(d), away from the region of the phase space dominated by $\mathrm{CC}$ processes.

\section{A.3 Tau channel control samples}

The description of narrow jets with at least one central high $P_{T}$ track as background to tau jets is checked by investigating tau-like jets satisfying the requirements given in Table 2, in the same $P_{T}$ and polar angle region as tau jets in the final sample. Such tau-like jets are selected in an event sample of inclusive NC events defined by an isolated electron in the phase space $P_{T}^{e}>10 \mathrm{GeV}, \theta_{e}<140^{\circ}$ and $D(e ;$ jet $)>1.0$. The polar angle distribution of the tau-like jet with the highest $P_{T}$ is shown in Fig. 13(a). To control any influence of lost or mismeasured energy on the hadronic final state and therefore on tau-like jets, $P_{T}^{\text {miss }}>12 \mathrm{GeV}$ is required in the inclusive $\mathrm{NC}$ event sample. A sample of dijets in photoproduction is also selected, mainly by requiring
$V_{\text {ap }} / V_{\mathrm{p}}>0.2, \delta_{\text {miss }}>10 \mathrm{GeV}$ and rejecting $\mathrm{NC}$ events with a scattered electron, in addition to requiring two jets with $P_{T}^{\text {jet1,jet2 }}>20,15 \mathrm{GeV}$. The polar angle distribution of the tau-like jet with the highest $P_{T}$ is shown in Fig. 13(b). The data are found to agree with the MC simulation within the total uncertainties in all of the above samples.

The total background in the final sample is controlled using the selection steps described in Table 2. Figure 13 shows the polar angle (c) and track multiplicity (d) distributions of tau-like jets in the CC-like sample, where all the isolation criteria of Table 2 are applied with the exception of the track isolation requirements. Good agreement between the data and the SM prediction is observed in these samples.

\section{References}

1. T. Ahmed et al. (H1 Collaboration), DESY preprint 94-248 (1994)

2. C. Adloff et al. (H1 Collaboration), Eur. Phys. J. C 5, 575 (1998). hep-ex/9806009

3. V. Andreev et al. (H1 Collaboration), Phys. Lett. B 561, 241 (2003). hep-ex/0301030

4. A. Aktas et al. (H1 Collaboration), Eur. Phys. J. C 33, 9 (2004). hep-ex/0310032

5. S. Chekanov et al. (ZEUS Collaboration), Phys. Lett. B 559, 153 (2003). hep-ex/0302010

6. S. Chekanov et al. (ZEUS Collaboration), DESY-03-188

7. A. Aktas et al. (H1 Collaboration), Phys. Lett. B 599, 159 (2004). hep-ex/0405070 
8. C. Diaconu, DESY-04-006,. hep-ph/0401111

9. T. Carli, D. Dannheim, L. Bellagamba, Mod. Phys. Lett. A 19, 1881 (2004). hep-ph/0402012

10. S.Y. Choi et al., Eur. Phys. J. C 51, 543 (2007). hep-ph/0612302

11. S.Y. Choi et al., Acta Phys. Pol. B 38, 3415 (2007). 0710.3334 [hep-ph]

12. S. Chekanov et al. (ZEUS Collaboration), 0807.0589 [hep-ex]

13. S. Chekanov et al. (ZEUS Collaboration), Phys. Lett. B 583, 41 (2004). hep-ex/0311028

14. A. Aktas et al. (H1 Collaboration), Eur. Phys. J. C 48, 699 (2006). hep-ex/0604022

15. U. Baur, J.A. Vermaseren, D. Zeppenfeld, Nucl. Phys. B 375, 3 (1992)

16. K.P. Diener, C. Schwanenberger, M. Spira, hep-ex/0302040

17. K.P. Diener, C. Schwanenberger, M. Spira, Eur. Phys. J. C 25, 405 (2002). hep-ph/0203269

18. P. Nason, R. Rückl, M. Spira, J. Phys. G 25, 1434 (1999). hep-ph/ 9902296

19. M. Spira, hep-ph/9905469

20. P. Aurenche et al., Z. Phys. C 56, 589 (1992)

21. H.L. Lai et al., Phys. Rev. D 55, 1280 (1997). hep-ph/9606399

22. C. Diaconu, Parton shower effects for $W$ production at HERA, in Proceedings of the Workshop "Monte Carlo Generators for HERA Physics", ed. by A.T. Doyle et al., DESY (1998), DESY-PROC1999-02, 631

23. H. Jung, RAPGAP version 3.1. Comput. Phys. Commun. 86, 147 (1995)

24. A. Kwiatkowski, H. Spiesberger, H.J. Möhring, Comput. Phys. Commun. 69, 155 (1992)

25. C. Berger, P. Kandel, A new generator for wide angle bremsstrahlung, in Proceedings of the Workshop "Monte Carlo Generators for HERA Physics", ed. by A.T. Doyle et al., DESY (1998), DESY-PROC-1999-02, p. 596

26. T. Sjöstrand, S. Mrenna, P. Skands, PYTHIA version 6.4. J. High Energy Phys. 0605, 026 (2006). hep-ph/0603175

27. C. Adloff et al. (H1 Collaboration), Eur. Phys. J. C 25, 13 (2002). hep-ex/0201006

28. A. Aktas et al. (H1 Collaboration), Phys. Lett. B 602, 14 (2004). hep-ex/0408044

29. G.A. Schuler, H. Spiesberger, DJANGOH version 1.4, Django: the interface for the event generators heracles and lepto, in Proceedings of the Workshop "Physics at HERA", vol. 3, ed. by W. Buchmüller and G. Ingelman, DESY (1991), p. 1419

30. L. Lönnblad, Comput. Phys. Commun. 71, 15 (1992)

31. T. Abe, GRAPE version 1.1. Comput. Phys. Commun. 136, 126 (2001). hep-ph/0012029

32. R. Brun et al., "GEANT3 user's guide", CERN-DD/EE/84-1

33. I. Abt et al. (H1 Collaboration), Nucl. Instrum. Methods A 386, 310 (1997)

34. I. Abt et al. (H1 Collaboration), Nucl. Instrum. Methods A 386, 348 (1997)

35. B. Andrieu et al. (H1 Calorimeter Group Collaboration), Nucl. Instrum. Methods A 336, 460 (1993)

36. B. Andrieu et al. (H1 Calorimeter Group Collaboration), Nucl. Instrum. Methods A 336, 499 (1993)

37. B. Andrieu et al. (H1 Calorimeter Group Collaboration), Nucl. Instrum. Methods A 350, 57 (1994)

38. R.D. Appuhn et al. (H1 SPACAL Group Collaboration), Nucl. Instrum. Methods A 386, 397 (1997)

39. C. Kleinwort, $\mathrm{H} 1$ alignment experience, in Proceedings of "First LHC Detector Alignment Workshop", ed. by S. Blusk et al., CERN (2006), CERN-2007-04, 41
40. C. Adloff et al. (H1 Collaboration), Eur. Phys. J. C 30, 1 (2003). hep-ex/0304003

41. P. Bruel, Recherche d'interactions au-delà du Modèle Standard à HERA. Ph.D. thesis, Université Paris (1998), available at http://www-h1.desy.de/psfiles/theses/

42. C. Adloff et al. (H1 Collaboration), Eur. Phys. J. C 13, 609 (2000). hep-ex/9908059

43. A. Aktas et al. (H1 Collaboration), Phys. Lett. B 583, 28 (2004). hep-ex/0311015

44. F.D. Aaron et al. (H1 Collaboration), Phys. Lett. B 668, 268 (2008). 0806.3987 [hep-ex]

45. M. Peez, Recherche de déviations au Model Standard dans les processus de grande énergie transverse sur le collisionneur électron-proton HERA. Ph.D. thesis, Université de Lyon (2003), DESY-THESIS-2003-023, available at http://www-h1.desy.de/ psfiles/theses/

46. B. Portheault, Première mesure des sections efficaces de courant chargé et neutre avec le faisceau de positrons polarisé à HERA II et analyses QCD-électrofaibles. Ph.D. thesis, Université Paris XI (2005), LAL-05-05, available at http://www-h1.desy.de/ psfiles/theses/

47. S.D. Ellis, D.E. Soper, Phys. Rev. D 48, 3160 (1993). hep-ph/ 9305266

48. S. Catani et al., Nucl. Phys. B 406, 187 (1993)

49. F. Jacquet, A. Blondel, Detectors for charged current events, in Proceedings of "Study of an ep Facility for Europe", ed. by U. Amaldi, DESY (1979), DESY 79/48, 391

50. D.M. South, Events with isolated leptons and missing transverse momentum in $e^{+} p$ collisions at HERA. Ph.D. thesis, University of Manchester (2003), DESY-THESIS-2003-030, available at http://www-h1.desy.de/psfiles/theses/

51. G. Brandt, Analysis of events with isolated leptons and missing transverse momentum in $e p$ collisions at HERA. Ph.D. thesis, University of Heidelberg (2007), available at http://www-h1. desy.de/psfiles/theses/

52. Y.R. de Boer, Measurement of single $W$ boson production in ep scattering. Ph.D. thesis, Institute for Theoretical and Experimental Physics, Moscow (2007), available at http://www-h1. desy.de/psfiles/theses/

53. W.M. Yao et al. (Particle Data Group), J. Phys. G 33, 1 (2006)

54. U. Baur, D. Zeppenfeld, Nucl. Phys. B 325, 253 (1989)

55. K. Hagiwara et al., Nucl. Phys. B 282, 253 (1987)

56. F. Herzog, Phys. Lett. B 148, 355 (1984) [Erratum: Phys. Lett. B 155, 468 (1985)]

57. J.C. Wallet, Phys. Rev. D 32, 813 (1985)

58. J.A. Grifols, S. Peris, J. Sola, Phys. Lett. B 197, 437 (1987) [Erratum: Phys. Lett. B 218, 508 (1989)]

59. M. Suzuki, Phys. Lett. B 153, 289 (1985)

60. J.J. Van Der Bij, Phys. Rev. D 35, 1088 (1987)

61. V.M. Abazov et al. (DØ Collaboration), Phys. Rev. D 71, 091108 (2005). hep-ex/0503048

62. T. Aaltonen et al. (CDF Collaboration), Phys. Rev. D 76, 111103 (2007). 0705.2247 [hep-ex]

63. S. Schael et al. (ALEPH Collaboration), Phys. Lett. B 614, 7 (2005)

64. J. Abdallah et al. (DELPHI Collaboration), Eur. Phys. J. C 54, 345 (2008). 0801.1235 [hep-ex]

65. P. Achard et al. (L3 Collaboration), Phys. Lett. B 586, 151 (2004). hep-ex/0402036

66. G. Abbiendi et al. (OPAL Collaboration), Eur. Phys. J. C 33, 463 (2004). hep-ex/0308067 